\title{
Comparison of the Implementation of Three Common Types of Coupled CFD-DEM Model for Simulating Soil Surface Erosion
}

\author{
Yuan Guo ${ }^{1}$ and Xiong (Bill) $\mathrm{Yu}^{2 *}$
}

\begin{abstract}
${ }^{1}$ Graduate Research Assistant, Department of Civil Engineering, Case Western Reserve University, Cleveland, OH, Email: yxg223@case.edu.

${ }^{2} *$ Professor, Department of Civil Engineering, Case Western Reserve University, Cleveland, OH, Phone: 216-368-6247, Email: xiong.yu@ case.edu, Corresponding Author.
\end{abstract}

\begin{abstract}
Soil erosion is a common process studied by soil science, environmental engineering, geotechnical engineering, coastal engineering, and many other fields. In the areas of hydraulic engineering, the geotechnics of soil erosion remains a high priority topic as the bridge scour is a common cause of bridge failures. Accurate predictions of scour depth and soil erosion rate remain challenging, due to the limitations of existing scaled experimental approach in fulfilling the hydrological and hydrodynamic similarity requirements. Computational model offers a promising alternative to further the microscale understanding of soil erosion which can help to develop engineering tools in practice. Computational model that couples Computational Fluid Dynamics (CFD) and Discrete Element Method (DEM) to
\end{abstract}


simulate the behaviors of fluid-solid systems is promising to advance the current tools for soil erosion analyses. Different mathematical forms for laminar fluid flows exist for the coupled CFD-DEM model as documented in published literatures and implemented in commercial and open-source software; each of them is based on certain physics assumptions and corresponding mathematical treatments. There are, however, no direct comparison of the results of CFD-DEM models based on these seemingly different mathematical formulations, which would help researchers to select the proper simulation tool. This study implemented coupled CFD-DEM models based on three most common types of mathematical formats used in the previous modeling work. The results of different CFD-DEM models are firstly validated by comparing the results of simulating the free settling of a particle in fluid. A case study is then designed to compare the models in simulating the surface erosion of cohesionless soil inside a pipe flow using laminar flow equations. Comparison indicates that for a relatively sparse particle-fluid system, the difference of the three models is negligible. For a dense particle-fluid system, simulation with the three different mathematical formats can predict different results (as large as $10 \%$ in the fluid velocity and $20 \%$ in the particle drag force for the simulation case study analyzed). The results of this case study indicate that the three CFD-DEM models achieved comparable results for simulating soil erosion from an engineering perspective, however, the differences between these models, which originate from their underlying physics assumptions, must be kept in mind in selecting an appropriate simulation model as well as in comparing the results from different models.

Keywords: Computational Fluid Dynamics (CFD), Discrete Element Method (DEM), CFDDEM, soil erosion, geotechnics of soil erosion, fluid-solid interaction 


\section{Introduction}

Bridge scour is a common issue in hydraulic and geotechnical engineering. It has also been found to be the number one cause for bridge failures (Briaud et al., 2001). Accurate predictions of the scour depth and erosion rate under turbulent flow remain challenging, especially for cohesive soils. A major obstacle in understanding the erosion process is the complex interactions between water and soil as well as those among soil particles. The erodibility of soil is controlled by its physical properties, geochemical properties, and also affected by the biological activities (Mena et al, 1999; Tejada and Gonzalez, 2006; Wynn et al., 2008; Grabowski et al., 2011; Dickhudt et al., 2011). Most of the current soil erosion models are based on the data from scaled laboratory experiments or in-situ observations. A simplified method in describing the erosion process is to consider fluid and soil separately (Sumer, 2007), where the erosion model is consisted of erosion criteria model and erosion rate model. Such simplified method, however, does not consider the particle-level soil-water interaction under different flow conditions, i.e. laminar flow versus turbulent flow, which can have a major influence on the soil erosion process.

Mesoscale models considering the fluid-solid interaction have been introduced to simulate the interactions of soils with flowing water and to understand the soil erosion mechanism. Many of these studies are extensions of the models for fluid-solid systems, which are firstly introduced to simulate the behavior of fluidized beds based on the concept of locally averaged variables (Anderson and Jackson, 1967, 1969). The locally averaged variables are advantageous in treating the discontinuous fluid-solid system as a continuous system with equivalent properties, which help to significantly increase the computational 
efficiency. The interactions between solid and fluid is modeled with the drag force model (e.g., Wen and Yu, 1966). Based on this concept, Two Fluid Model (TFM) has been developed to simulate the fluid-solid interaction systems and is widely used in fields such as chemical and mechanical engineering (i.e., Monahan and Fox 2007; Bravo et al., 2007; Nguyen et al., 2009; Huang et al., 2010; Verma et al., 2013; Wang et al., 2014). This Eulerian-Eulerian model uses similar equation forms for the fluid phase and solid phase respectively based on volume fraction, and exchanges momentum between fluid and solid phases during the time-dependent calculation. The flow velocity and pressure in the NavierStokes equations are averaged by the porosity. To explain the influence of turbulence in particle-gas suspension, turbulence models, e.g., the $k-\varepsilon$ turbulence model, have also been implemented into TFM framework (Shah et al., 2013; Patro and Dash, 2014).

For granular materials such as sand, the physical and mechanical properties can be quite different from those of continuous materials. A physics based approach to describe granular materials is the Discrete Element Method (DEM) proposed by Cundall (1979). By using large amount of discrete particles, the DEM model is able to describe particle flows under Newton's Laws of Motion. In the research of fluidized beds, Tsuji (1992, 1993) proposed a coupling Computational Fluid Dynamics (CFD) model and DEM model to describe fluid-solid systems and demonstrated its advantages in predicting the fluid-solid interactions reasonably well. As an extension of TFM, the coupled CFD-DEM model combines the advantages of high efficiency in solving continuous fluid field by way of TFM and the realistic description of motion of discontinuous particle phase by DEM. It is therefore able to explain the behaviors of fluid-solid systems in the mesoscale, which are consistent with what observed by other researchers analyzing different types of applications (Zhu et al., 2007; Chen and Wang, 2014; Patil et al., 2015). This CFD-DEM model has also been used in geotechnical engineering to explain the complicated interactions between soil 
and fluid, e.g., debris flow, poromechanics, etc. (Zhao and Shan, 2013, 2014; Chen et al., 2011; Zhao et al., 2014).

Literature review on the CFD-DEM method has shown that there exist a few different mathematical forms for this Eulerian-Lagrangian model (Bouillard et al., 1989; Feng and Yu, 2004a). The major difference of these mathematical forms lies in the assumption for pressure exchange between fluid phase and solid phase, which is expressed as different pressure terms in the locally averaged Navier-Stokes equations. For the modeling of fluidized beds, it was believed that the variances in results due to different mathematical forms can be high and should not be ignored (Zhu et al., 2007). However, there has been no direct comparison of the results based on implementing different mathematical forms of CFD-DEM models.

This study aims to provide a direct comparison of the simulation results by three most common types of governing equations of the laminar CFD-DEM models. The results of different CFD-DEM model implementations are firstly validated by comparing with the standard experiment of free settling of a single particle in fluid. A testbed comparison case was then designed to compare three different CFD-DEM implementations on simulating soil erosion under pipe flow. It is assumed that sand particles, which is deposited inside a pipe under gravity, is subjected to pipe flow erosion. The results by different CFD-DEM simulation models are compared.

\section{Mathematical Formulations}

The mathematical formulations describing the physics and mechanical basis of the CFDDEM model are summarized in this section. Overall the fluid phase is described with the Navier-Stokes equations in Eulerian form; the solid phase is solved by Newton's Laws of Motion in Lagrangian form. Interactions between water and soil particles are calculated 
through a drag force model. During each coupling time step, fluid and particles will exchange data about their current statuses and update accordingly. The equation forms for particle and fluid phases in implementing the CFD-DEM models are described and compared in the following context.

\subsection{Governing equations for the fluid phase}

The Navier-Stokes equations have been averaged according to the volume fraction of fluid phase in the fluid-solid system. By adopting the concepts of locally averaged velocity and pressure, the fluid field is regarded as continuous in space even inside solid particles (Anderson and Jackson, 1967). The conservation of mass is described in Eq. (1). Three common mathematical formats for momentum conservation of the fluid, which are used in different CFD-DEM implementations, are listed in Eqs. (2a), (2b), and (2c).

$$
\begin{aligned}
& \frac{\partial \mathrm{n} \rho}{\partial \mathrm{t}}+(\nabla \cdot \mathrm{n} \rho \mathbf{u})=0 \\
& \frac{\partial \mathrm{n} \rho \mathbf{u}}{\partial \mathrm{t}}+(\nabla \cdot \mathrm{n} \rho \mathbf{u} \mathbf{u})=-\mathrm{n} \nabla \mathrm{p}+\mathrm{n} \nabla \cdot \mathbf{K}+\mathrm{n} \rho \mathbf{g}+\mathbf{f}^{\mathrm{p}} \\
& \frac{\partial \mathrm{n} \rho \mathbf{u}}{\partial \mathrm{t}}+(\nabla \cdot \mathrm{n} \rho \mathbf{u u})=-\mathrm{n} \nabla \mathrm{p}+\nabla \cdot \mathrm{n} \mathbf{K}+\mathrm{n} \rho \mathbf{g}+\mathbf{f}^{\mathrm{p}} \\
& \frac{\partial \mathrm{n} \rho \mathbf{u}}{\partial \mathrm{t}}+(\nabla \cdot \mathrm{n} \rho \mathbf{u u})=-\nabla \mathrm{p}+\nabla \cdot \mathrm{n} \mathbf{K}+\mathrm{n} \rho \mathbf{g}+\mathbf{f}^{\mathrm{p}}
\end{aligned}
$$

where, $\mathrm{n}$ is porosity from the solid phase; $\mathrm{t}$ is the time; $\mathbf{u}$ is the fluid velocity vector; $\rho$ is the density of fluid; $\mathrm{p}$ is fluid pressure; $\mathbf{K}$ is stress tensor of the fluid field, and $\mathbf{K}=$ $\mu\left(\nabla \mathbf{u}+(\nabla \mathbf{u})^{\mathrm{T}}\right)$ for laminar flow; $\mu$ is the fluid dynamic viscosity; $\mathbf{g}$ is gravitational acceleration and $\mathrm{g}_{\mathrm{z}}$ equals to $-9.80 \mathrm{~m} / \mathrm{s}^{2} ; \mathbf{f}^{\mathrm{p}}$ is volumetric fluid-particle interaction force, 
which equals to total concentrated interaction force (drag force \& buoyant force) divided by the volume of the mesh element.

These three mathematical forms are based on different physical assumptions and have been used by different researchers (i.e., Eq. (2a) by Chen et al., 2011; Zhao et al., 2014; Eq. (2b) by Zhao and Shan, 2013, 2014; Tomac and Gutierrez, 2014; Guo et al., 2014; Eq. (2c) by Feng and Yu, 2004a; Jovanovic et al., 2014). Besides, different commercial or opensource software use different formats of these equations (i.e., PFC3D Coarse-Grid Fluid Scheme with Eq. (2a); OpenFoam-LIGGGHTS with Eq. (2b)). Computational models based on these different formats are referred as Model A, B, C respectively in this paper, which is also summarized in Table 1.

The major difference of these formats lies in the pressure and stress tensor terms in the momentum equation. In Model $\mathrm{A}$, the momentum equation is normalized by extracting the porosity, leaving a volume averaged fluid-solid interaction force. The normalized equation has the similar form as the Navier-Stokes equation except for the body force term, which can simplify the numerical implementations. Model B assumes the pressure drop is shared by the fluid phase as well as the solid phase. So both the pressure term and the stress tensor term have been averaged by porosity. In contrast, Model $\mathrm{C}$ assumes only the fluid phase shares the pressure drop and there is no averaged porosity in front of the pressure gradient (Gidaspow, 1994; Jackson, 2000). Previous research shows Model B and Model C give similar results for fluid-solid system with mono-sized solid particles. However, for binary mixtures of particles, it was projected that there can be a significant difference between Model B and Model C (Zhu et al., 2007).

For a uniformly dispersed fluid-solid system, the porosity approximates to be a constant in space. Under this condition, the momentum equation in Model B can be simplified into the same form as that in Model A. However, as the spatial gradient of 
porosity increases, the mathematical difference between Model A and Model B increases. The porosity of soils depends on the packing density, particle size distribution, shape of particles, etc. Near the soil surface, the porosity variation increases. These factors may cause simulating errors by Model A. Also from Table 1, since the magnitude of porosity cannot be larger than one, the pressure gradient in Model $\mathrm{C}$ should be smaller compared with that in Models A and B. These observations are verified by the simulation study, which is described in the following context.

2.2. Governing equations for the particle phase

The behaviors of particles are simulated with the Discrete Element Method (DEM). In the DEM model, the particles are governed by Newton's Laws of Motion. The conservations of momentum and angular momentum for each particle read:

$$
\begin{aligned}
& \mathrm{m}_{i} \frac{\mathrm{d}_{i}^{p}}{\mathrm{dt}}=\sum_{\mathrm{j}=1}^{\mathrm{n}_{i}^{\mathrm{c}}} \mathbf{F}_{i j}^{\mathrm{c}}+\mathbf{F}_{i}^{f}+\mathbf{F}_{i}^{g} \\
& \mathrm{I}_{i} \frac{\mathrm{d} \mathbf{w}_{i}}{\mathrm{dt}}=\sum_{\mathrm{j}=1}^{\mathrm{n}_{i}^{\mathrm{c}}} \mathbf{M}_{i j}
\end{aligned}
$$

where, $\mathrm{m}_{i}$ is the mass of particle $i$; $\mathbf{U}_{i}^{p}$ is the translational velocity of particle $i$; $\mathrm{t}$ is the time; $\mathrm{n}_{i}^{c}$ is the number of total contacts of particle $i ; \mathbf{F}_{i j}^{c}$ is the contact force acting on particle $i$ from particle $j ; \mathbf{F}_{i}^{f}$ is the fluid interaction force acting on particle $i$, which has the same magnitude but opposite direction as $\boldsymbol{f}^{p} ; \mathbf{F}_{i}^{g}$ is the gravitational force of particle $i$; $\mathrm{I}_{i}$ is the moment of inertia of particle $i ; \boldsymbol{w}_{i}$ is the angular velocity of particle $i ; \mathbf{M}_{i j}$ is the torque applied from particle $j$ to particle $i$. 
The constitutive model, which describes the interactions between two contacting particles, is schematically shown in Figure 1: there are spring and dashpot models in the normal and tangential directions respectively, and a sliding friction model in the tangential direction. In DEM software such as PFC3D, it requires to set local damping and viscous damping between particles. The local damping is directly applied into the momentum equations and the viscous damping is considered as a dashpot in contacts (dashpots in Figure 1 belong to viscous damping). The local damping was used to help to achieve the initial gravitational balance during the DEM simulations. In the subsequent CFD-DEM coupling computation, both the viscous damping ratio and the local damping ratio are set as zero for simplifications.

\subsection{Fluid-solid interaction force}

Interaction forces between water and soil particles include pressure gradient force, drag force, virtual mass force, lifting force, capillary force, etc. (Zhu et al., 2007). This study assumes a fully saturated condition where the soil grains are completely submerged under water. Therefore the capillary force is set as zero. The virtual mass force and lifting force are small compared with the drag force and are therefore neglected in the CFD-DEM model implemented in this paper. The pressure gradient force is simplified to the buoyant force acting upon each particle.

There are different fluid-solid interaction force models for the fluid-solid systems. The Di Felice drag force model (Di Felice, 1994) has been used in this paper as is shown in Eq. (5). Based on different pressure drop assumptions, the drag forces of Model A, Model B and Model C should follow the relationship of Eq. (6) (Gidaspow, 1994; Feng and Yu, 2004b). This paper focuses on comparing the three different forms of governing equations 
numerically. Therefore the same fluid-particle interaction force is used (Eq. (5)) for the three models so that the differences in the simulation results are primarily due to the different formats of governing equations. The drag coefficient is calculated through the Wen and Yu's equation (Wen and Yu, 1966, see Eq. (7)).

$\mathbf{F}_{i}^{\mathrm{f}}=\frac{1}{8} \mathrm{C}_{\mathrm{D}} \pi \rho_{f} \mathrm{~d}_{p}^{2}|\mathbf{u}-\mathbf{U}|(\mathbf{u}-\mathbf{U}) n^{1-\chi}$

$\mathbf{F}_{i}^{\mathrm{f}, \mathrm{a}}=\mathbf{F}_{i}^{\mathrm{f}, \mathrm{b}}=n \mathbf{F}_{i}^{\mathrm{f}, \mathrm{c}}$

where, $\mathbf{F}_{i}^{\mathrm{f}, \mathrm{a}}, \mathbf{F}_{i}^{\mathrm{f}, \mathrm{b}}$ and $\mathbf{F}_{i}^{\mathrm{f}, \mathrm{c}}$ are the drag forces acting on particle $i$ of Model A, Model B and Model $\mathrm{C} ; \mathrm{C}_{\mathrm{D}}$ is the drag coefficient; $\rho_{f}$ is the fluid density; $d_{p}$ is the particle diameter; $\mathbf{u}, \mathbf{U}$ are the fluid velocity and particle velocity. The drag coefficient is calculated by Eqs. (7) to (9).

$\mathrm{C}_{\mathrm{D}}=\frac{24}{R_{e}}\left(1+0.15 R_{e}^{0.687}\right) \quad$ for $R_{e} \leq 1000$

$\mathrm{C}_{\mathrm{D}}=0.44 \quad$ for $R_{e}>1000$

$R_{e}=\frac{n \rho_{f}|\boldsymbol{u}-\boldsymbol{U}| d_{p}}{\mu}$

$\chi=3.7-0.65 \exp \left(-\frac{\left(1.5-\log _{10} R_{e}\right)^{2}}{2}\right)$

where, $R_{e}$ is the particle Reynolds number.

\section{Implementation of Coupled CFD-DEM Model}

The coupled CFD-DEM framework is implemented by developing customized code under Matlab environment, which links the fluid and particle modules provided in two commercial 
software, COMSOL for the CFD simulation and PFC3D for the particle simulation. The COMSOL module is based on the weak form of the partial differential equations. The Matlab code provides the main controller for data extraction, process manipulation, and information exchange between CFD and DEM modules. The flow chart of implementing the coupled CFD-DEM simulations is shown in Figure 2. For each coupling time step, the CFD and DEM modules operate separately with their respective initial conditions. The particle coordinates and porosity will be extracted from the results of DEM module simulation and transmitted to CFD module. The drag force and flow velocity from the results of CFD simulation are extracted and applied to the DEM model. These input will drive the CFD and DEM models to calculate the phase behaviors at the next time step. The process continues until the condition for termination reaches, i.e., by the total length of simulation time.

The size of coupling time step is determined by a sensitivity study, which is a comprehensive consideration of computational efficiency and accuracy. Based on the results of sensitivity analyses on the time step, the time step for CFD is set to be $10^{-4}$ seconds, which is much larger than the time step for DEM which is in the range of $10^{-6}$ second. The CFD module and DEM module use different time steps during the calculation to ensure their respective stability and accuracy. The CFD and DEM modules are set to exchange data and therefore couple at every $10^{-3}$ seconds. In general, the choice of coupling time step is dependent upon the balanced consideration of computational time and accuracy requirements. For example, for the commonly used open-source code OpenFoam-LIGGGHTS, the coupling step can be set as small as $10^{-4}$ seconds.

\section{Comparison of a Single Particle-Fluid System}


The interaction between fluid and particles is firstly compared through simulating the free settling of a particle in fluid, i.e., sand particle in water is released and settles freely. The sand particle is idealized as a sphere. Once being released, the damping force from water will increase as the particle accelerates and finally reaches a balance against gravity with a constant settling speed. With the same drag force model, the settling velocity is mainly determined by the particle's properties, e.g., density, diameter, etc., and its surrounding fluid velocity. The simulation model is shown in Figure 3: a spherical particle with a density of $2.65 \mathrm{~g} / \mathrm{cm}^{3}$ and a diameter of $2.0 \mathrm{~mm}$ is released in a cuboid tank filled with water. The water tank has a dimension of $0.1 \mathrm{~m} \times 0.1 \mathrm{~m} \times 0.3 \mathrm{~m}$.

The simulated particle velocities versus time by different models are compared in Figure 4. The final settling velocities in the three different CFD-DEM implementations are similar. The close match of simulation results in the three models indicates that the coupled CFD-DEM models are properly implemented. The settling velocities predicted by Model A and Model B are almost identical $(0.1550 \mathrm{~m} / \mathrm{s})$ since the variance of porosity is small. While the final settling velocity by Model $\mathrm{C}$ is slightly lower $(0.1548 \mathrm{~m} / \mathrm{s}$ or $0.13 \%$ lower $)$, indicating a slightly smaller fluid velocity surrounding the particle. The results imply that for fluid with sparse particle phase, the differences in the fluid-solid interaction forces by these three models are small.

\section{Model Description of a Dense Particle-Fluid System}

A three-dimensional soil erosion model are built to compare the performance of these three models in a dense particle-fluid system. It also provides an opportunity to analyze the erosion behavior of sands in the mesoscale. Sand grains were firstly generated and deposited inside a pipe under gravity, and then subjected to the erosion of flowing water. The pipe is 
assumed to have a length of $0.6 \mathrm{~m}$ and a diameter of $0.1 \mathrm{~m}$, as is shown in Figure 5. Non-slip boundaries are used for the pipe walls in the CFD model. The inlet (left) is assumed to maintain a constant flow velocity of $1.0 \mathrm{~m} / \mathrm{s}$, and the outlet (right) is assumed to have a constant pressure boundary of $0.0 \mathrm{~Pa}$.

Totally 9375 mono-sized particles with diameters of $2.0 \mathrm{~mm}$ are generated at location from $0.10 \mathrm{~m}$ to $0.15 \mathrm{~m}$ near the inlet, and the CFD model consists of 10829 tetrahedral elements. Parameters for the sand particles are derived from the inverse analysis through triaxial test simulation, and the results are listed in Table 2. These microscale parameters of sand particles characterize an internal friction angle of 26 degree. The same initial conditions and boundary conditions are applied during the implementation of the three types of mathematical formats of CFD-DEM model. Totally 5.0 seconds of soil erosion are simulated.

\section{Results and Discussions}

The three different coupled CFD-DEM models are implemented following the procedures described in Section 3. The results show that after 5.0 seconds flow erosion, the sand profiles in Model A, Model B and Model C become relatively stable.

\subsection{Overall velocity and porosity distribution}

The fluid and particle velocities at 5.0 seconds are shown in Figure 6. Overall, the velocity field and shape of sand pile at 5.0s are similar. The maximum fluid velocity at 5.0s predicted by the three models are close to each other. The maximum particle velocity of the solid phase is the highest in Model C, followed by Models B and A. As described in the early context of this paper, a major difference between the three CFD-DEM models are the 
treatment of the fluid pressure and porosity gradient. The porosity distributions of the sandpile at 5.0 seconds are plotted in Figure 7. The porosity is 1.0 in the free fluid field. The interactions of fluid and sand pile cause a non-uniform distribution of soil porosity. Overall, the internal zone of the sand pile is not significant affected. The closer to the surface of the sand pile, the larger the porosity. These observations are consistent with the macroscopic erosion process commonly observed.

6.2 Flow velocity and pressure field

Figure 8 shows the flow velocity distribution along the center line of cross section at $\mathrm{X}=$ $0.125 \mathrm{~m}$. Compared with a pure pile flow, the overall velocity profile shifted upwards under the influence of sand pile. The velocity profiles of Models A and B are close to each other, while for Model C it shows appreciable difference, particularly at the bottom part that intercepts into soils. Comparison between Model A and Model B shows that the spatial variation of porosity has a minor influence on the flow velocity in this case. Comparison between Model $\mathrm{C}$ and Model $\mathrm{A} / \mathrm{B}$ implies that the effects of treating the pressure as pore pressure (only shared by the fluid phase) can lead to appreciable difference in flow velocity, particularly for areas with relatively low porosity.

Figure 9 shows the evolvement of fluid velocity at the surface of sandpile (height $=$ $0.02 \mathrm{~m})$. The velocity processes are different among different models. At the end of 5.0s, the velocity of Model A and Model B has a difference up to $1.5 \%$. For Model $\mathrm{C}$, the fluid velocity in the particle zone is around $10 \%$ higher than those of Models A and B. This will lead to a relatively large drag force predicted by Model $\mathrm{C}$ and consequently a higher rate of erosion. 
The fluid pressure contour at 5.0s is show in Figure 10. The pressure fields in Model A and Model B are similar both in the fluid zone and inside the particle zone. In Model C, the pressure gradient in the particle zone is smaller than that in Model A or B, which has been discussed mathematically as resulting from the different pressure gradient term in Eq. (2c). Figure 11 shows the pressure field along the center line of the cross section at the $\mathrm{X}$ coordinate of $0.125 \mathrm{~m}$. The time processes of fluid pressure above the soil surface by these three models are plotted in Figure 12. The comparison indicates that the pressure processes are similar as predicted by the three CFD-DEM models.

6.3 Particle movement, erosion rate and kinetic energy

Figure 13 compares the sand profile before and after flow erosion simulated with three types of models. After the 5.0-second flow erosion, sandpile reaches a steady erosion profile. As discussed previously, mathematically Model A and B can be similar when the spatial variance of porosity is small, which means the major difference between Model A and Model B should be around the fluid-solid interface. Such difference is found to be very small in the case simulated here. Model $\mathrm{C}$ predicts a relatively large flow velocity on the soil surface and therefore a larger drag force; the final sand profile shows more erosion than those predicted by Models A and B.

The contour graph of drag force (exclude buoyant force) acting on soil particles at time $=5.0$ seconds is shown in Figure 14. Drag force on the upwind side (left side) is much larger than that on the downwind side. Model C predicts a relatively large magnitude of drag force than Model A/B. Inside the sandpile, flow velocity decreases and drag force is smaller than $2.5 \times 10^{-4} \mathrm{~N}$. The distribution of drag force shows erosion firstly occurs at the upper corner of the upstream slope, where there exists a larger magnitude of fluid flow. The 
particle velocity distribution at time $=5.0$ seconds is given in Figure 15 (particles washed out from the pipe are not included). In Figure 15, the cross area of each curve represents the percentage of particles within a certain velocity range. Velocity distribution curves of Model A and Model B are similar and slightly flatter than that of Model C, which is consistent with the observed behaviors of the particle drag force.

The total kinetic energy of the solid phase are calculated. Figure 16, 17 and 18 compare the translational kinetic energy, rotational kinetic energy, and the total kinetic energy of soil particles of different CFD-DEM models. For particles washed away from the pipe, the translational and rotational velocities are recorded and included. These figures show that translational motion energy is the major part of the total kinetic energy, and only less than $5 \%$ of kinetic energy come from rotational motion. However this may not fully reflect the real erosion process since the current drag force model has ignored the angular momentum transferred from fluid to particles. Sliding friction and particle collision are two major mechanism for the energy dissipation. Overall, the erosion rate predicted by the CFDDEM models follow the following sequence:

Erosion Rate: $\quad$ Model $\mathrm{B} \approx$ Model $\mathrm{A}<$ Model $C$

\subsection{Overall performance of different CFD-DEM models}

Comparison among the simulation cases implemented in this study shows Model A and Model B give similar predictions for both fluid and particle phases. Model $\mathrm{C}$ predicts relatively higher flow velocity in the particle zone (10\% higher than those of Models A and B) and larger average drag force (around 20\% larger than those by Models A and B). After the 5.0-second flow erosion, the final shape of steady profiles of sandpile in Model A, Model B and Model $\mathrm{C}$ are similar visually (Figure 13). Using the number of particles passing the right 
boundary of sand pile $(\mathrm{X}=0.18 \mathrm{~m})$ as the determinant criteria for erosive particles (Figure 13a), then 679 sand particles have been eroded in Model $\mathrm{C}$ during the five seconds erosion, while in Model A and Model B, this number is 442 and 396 respectively. With this criteria, erosion rate by Model $\mathrm{C}$ is $54 \%$ higher than that in Model $\mathrm{A}$, and is $71 \%$ higher compared with Model B.

Overall these simulation cases show that the variation of pressure and velocity of fluid and particle phases due to spatial variation of porosity can have different extents of influence on the results of CFD-DEM models. For sparse particle-fluid systems, this difference is negligible. However for dense particle-fluid systems, Model C tends to predict a larger drag force and a smaller pressure variance based on its physics assumption that the pore space shares the total pressure drop. From the computational efficiency perspective, these three models were found to have similar computational performances. For the simulation of 5.0second of soil erosion as in this study, solving each of these three models takes around 100 hours with a desktop computer of a $3.40 \mathrm{GHz}$ processor.

\section{Conclusion and Recommendation}

This paper compares the mathematical forms of three types of CFD-DEM modeling frameworks that are commonly used by researchers and open-source or commercial software. It discussed the physical basis of these three models and highlighted the differences of these models in the treatment of fluid momentum equation. By developing customized code to link two commercial software, COMSOL and PFC3D, these three models have been implemented and compared in the modeling of sparse and dense particle-fluid systems. Two simulation cases are compared, which include the free settling of a sand particle and the erosion behavior of sands subjected to pipe flow. Comparison of the simulation results indicates that for relatively sparse particle-fluid systems, the difference of three models at sand scale is negligible. However for dense particle-fluid systems, the different treatment of pressure (pore pressure shared by fluid or overall pressure shared by fluid and particles) and volume 
fraction (porosity distribution) can lead to deviations in the simulation results. The extent of influence is decided by the extent of deviation of the assumptions to the actual fluid particle system. Overall, the CFD-DEM model describes fluid-solid interactions accurately in mesoscale and is able to provide insight into soil erosion mechanism. The major difference of three CFD-DEM models lies in their simplified treatment based on assumed spatial and temporal distribution of fluid pressure and particle porosity. Such physical assumptions on fluid pressure and porosity of different CFD-DEM models need to be kept in mind when selecting or comparing open-source or commercial code.

\section{Acknowledgements}

This work is partially supported by the US National Science Foundation via Grant No. 0900401. 


\section{References}

[1] J.L. Briaud, F.C.K. Ting, H.C. Chen, Y. Cao, S.W. Han, K.W. Kwak, Erosion function apparatus for scour rate predictions, Journal of Geotechnical and Geoenvironmental Engineering 127(2) (2001) 105-113.

[2] M. Martinez-Mena, L.K. Deeks, A.G. Williams, An evaluation of a fragmentation fractal dimension technique to determine soil erodibility, Geoderma 90 (1999) 87-98.

[3] M. Tejada, J.L. Gonzalez, The relationships between erodibility and erosion in a soil treated with two organic amendments, Soil and Tillage Research 91 (2006) 186-198.

[4] T.M. Wynn, M.B. Henderson, D.H. Vaughan, Changes in streambank erodibility and critical shear stress due to subaerial processes along a headwater stream, southwestern Virginia, USA, Geomorphology 97 (2008) 260-273.

[5] R.C. Grabowski, I.G. Droppo, G. Wharton, Erodibility of cohesive sediment: the importance of sediment properties, Earth-Science Reviews 105(3-4) (2011) 101-120.

[6] P.J. Dickhudt, C.T. Friedrichs, L.P. Sanford, Mud matrix solids fraction and bed erodibility in the York River estuary, USA, and other muddy environments, Continental Shelf Research 31 (2011) S3-S13.

[7] J.C. Santamarina, Soil behavior at the microscale: particle forces, Paper presented at the Proc. Symp. Soil Behavior and Soft Ground Construction (2001).

[8] J.L. Briaud, Scour depth at bridges: method including soil properties. I: maximum scour depth prediction, Journal of Geotechnical and Geoenvironmental Engineering 13 (2015) 04014104.

[9] J.L. Briaud, Scour depth at bridges: method including soil properties. II: time rate of scour prediction, Journal of Geotechnical and Geoenvironmental Engineering 13 (2015) 04014105.

[10] B.M. Sumer, Mathematical modelling of scour: a review, Journal of Hydraulic Research 45(6) (2007) 723-735.

[11] T.B. Anderson, R. Jackson, A fluid mechanical description of fluidized beds: equations of motion, I\&EC Fundamentals 6(4) (1967) 527-539. 
[12] T.B. Anderson, R. Jackson, A fluid mechanical description of fluidized beds: comparison of theory and experiment, I\&EC Fundamentals 8(1) (1969) 137-144.

[13] S.M. Monahan, R.O. Fox, Linear stability analysis of a two-fluid model for air-water bubble columns, Chemical Engineering Science 62(12) (2007) 3159-3177.

[14] H.R. Bravo, J. S. Gulliver, M. Hondzo, Development of a commercial code-based two-fluid model for bubble plumes, Environmental Modelling \& Software 22(4) (2007) 536-547.

[15] K.D. Nguyen, S. Guillou, J. Chauchat, N. Barbry, A two-phase numerical model for suspended-sediment transport in estuaries, Advances in Water Resources 32(8) (2009) 11871196.

[16] Q. Huang, C. Yang, G. Yu, Z.S. Mao, CFD simulation of hydrodynamics and mass transfer in an internal airlift loop reactor using a steady two-fluid model, Chemical Engineering Science 65(20) (2010) 5527-5536.

[17] V. Verma, N.G. Deen, J.T. Padding, J.A.M. Kuipers, Two-fluid modeling of threedimensional cylindrical gas-solid fluidized beds using the kinetic theory of granular flow, Chemical Engineering Science 102 (2013) 227-245.

[18] S. Wang, L. Zhao, C. Wang, Y. Liu, J. Gao, Y. Liu, Q. Cheng, Numerical simulation of gassolid flow with two fluid model in a spouted-fluid bed, Particuology 14 (2014) 109-116.

[19] S. Shah, J. Ritvanen, T. Hyppanen, S. Kallio, Wall effects on space averaged two-fluid model equations for simulations of gas-solid flows in risers, Chemical Engineering Science 89 (2013) 206-215.

[20] P. Patro, S.K. Dash, Two-fluid modeling of turbulent particle-gas suspensions in vertical pipes, Powder Technology 264 (2014) 320-331.

[21] P.A. Cundall, O.D.L. Strack, A discrete numerical model for granular assemblies, Geotechnique 29 (1979) 47-65.

[22] Y. Tsuji, T. Tanaka, T. Ishida, Lagrangian numerical simulation of plug flow of cohesionless particles in a horizontal pipe, Powder Technology 71 (1992) 239-250.

[23] Y. Tsuji, T. Kawaguchi, T. Tanaka, Discrete particle simulation of two dimensional fluidized bed, Powder Technology 77 (1993) 79-87. 
[24] D. Gidaspow, Multiphase Flow and Fluidization: Continuum and Kinetic Theory Descriptions, Academic Press, 1994.

[25] R. Jackson, The Dynamics of Fluidized Particles, Cambridge University Press, 2000.

[26] H.P. Zhu, Z.Y. Zhou, R.Y. Yang, A.B. Yu, Discrete particle simulation of particulate systems: Theoretical developments, Chemical Engineering Science 62(13) (2007) 3378-3396.

[27] X. Chen, J. Wang, A comparison of two-fluid model, dense discrete particle model and CFD-DEM method for modeling impinging gas-solid flows, Powder Technology 254 (2014) 94-102.

[28] A.V. Patil, E.A.J.F. Peters, J.A.M. Kuipers, Comparison of CFD-DEM heat transfer simulations with infrared/visual measurements, Chemical Engineering Journal 277 (2015) $388-401$.

[29] J. Zhao, T. Shan, Coupled CFD-DEM simulation of fluid-particle interaction in geomechanics, Powder Technology 239 (2013) 248-258.

[30] T. Shan, J. Zhao, A coupled CFD-DEM analysis of granular flow impacting on a water reservoir, Acta Mechanica 225(8) (2014) 2449-2470.

[31] , J.X. Bouillard, R.W. Lyczkowski, D. Gidaspow, Porosity distributions in a fluidized bed with an immersed obstable, A.I.Ch.E. Journal 35(6) (1989) 908-922.

[32] Y.Q. Feng, A.B. Yu, Assessment of model formulations in the discrete particle simulation of gas-solid flow. Ind. Eng. Chem. Res. 43 (2004a) 8378-8390.

[33] F. Chen, E.C. Drumm, G. Guiochon, Coupled discrete element and finite volume solution of two classical soil mechanics problems, Computers and Geotechnics 38(5) (2011) 638-647.

[34] T. Zhao, G.T. Houlsby, S. Utili, Investigation of granular batch sedimentation via DEMCFD coupling, Granular Matter 16(6) (2014) 921-932.

[35] I. Tomac, M. Gutierrez, Fluid lubrication effects on particle flow and transport in a channel, International Journal of Multiphase Flow 65 (2014) 143-156.

[36] L. Guo, K. Morita, Y. Tobita, Numerical simulation of gas-solid fluidized beds by coupling a fluid-dynamics model with the discrete element method. Annals of Nuclear Energy 72 (2014) 31-38. 
[37] A. Jovanovic, M. Pezo, L. Pezo, L. Levic, DEM/CFD Analysis of Granular Flow in Static Mixers, Powder Technology 266 (2014) 240-248.

[38] R. Di Felice, The voidage function for fluid-particle interaction systems, Int. J. Multiphase Flow 20(1) (1994) 153-159.

[39] Y.Q. Feng, A.B. Yu, Assessment of model formulations in the discrete particle simulation of gas-solid flow. Industrial \& Engineering Chemistry Research 43 (2004b) 8378-8390.

[40] C.Y. Wen, Y.H. Yu, Mechanics of fluidization, Chemical Engineering Progress Symposium Series 62 (1966) 100-111. 


\section{List of Tables:}

Table 1. Comparison of different equation forms for the fluid phase.

Table 2. Parameters used in the CFD-DEM model. 
Table 1. Comparison of different equation forms for the fluid phase.

\begin{tabular}{lc}
\hline \multicolumn{1}{c}{ Models } & Basic Equations \\
$\begin{array}{l}\text { Model A (Chen et al., } \\
\text { 2011; Zhao et al., 2014) }\end{array}$ & $\frac{\partial \mathrm{n} \rho}{\partial \mathrm{t} \rho \mathbf{u}}+(\nabla \cdot \mathrm{n} \rho \mathbf{u})=0$ \\
& $\frac{\partial \mathrm{n} \rho}{\partial \mathrm{t}}+(\nabla \cdot \mathrm{n} \rho \mathbf{u})=0$ \\
\hline Model B (Zhao and Shan, & $\frac{\partial \mathrm{n} \rho \mathbf{u}}{\partial \mathrm{t}}+(\nabla \cdot \mathrm{n} \rho \mathbf{u u})=-\mathrm{n} \nabla \mathrm{p}+\nabla \cdot \mathrm{n} \mathbf{K}+\mathrm{n} \rho \mathbf{g}+\mathbf{f}^{\mathrm{p}}$ \\
2013, 2014; Tomac and & \\
Gutierrez, 2014; Guo et & \\
al., 2014) & $\frac{\partial \mathrm{n} \rho}{\partial \mathrm{t}}+(\nabla \cdot \mathrm{n} \rho \mathbf{u})=0$ \\
\hline Model C (Feng and Yu, & $\frac{\partial \mathrm{n} \rho \mathbf{u}}{\partial \mathrm{t}}+(\nabla \cdot \mathrm{n} \rho \mathbf{u u})=-\nabla \mathrm{p}+\nabla \cdot \mathrm{n} \mathbf{K}+\mathrm{n} \rho \mathbf{g}+\mathbf{f}^{\mathrm{p}}$ \\
2004a; Jovanovic et al., \\
2014)
\end{tabular}


Table 2. Parameters used in the CFD-DEM model.

\begin{tabular}{ll}
\hline Parameter & Value \\
\hline Particle diameter & $2.0 \mathrm{~mm}$ \\
Particle density & $2600 \mathrm{~kg} / \mathrm{m}^{3}$ \\
Normal stiffness & $1.0 \mathrm{E} 8 \mathrm{~N} / \mathrm{m}$ \\
Shear stiffness & $1.0 \mathrm{E} 8 \mathrm{~N} / \mathrm{m}$ \\
Particle friction coefficient & 0.4 \\
Particle-wall friction coefficient & 0.1 \\
Fluid density & $1000 \mathrm{~kg} / \mathrm{m}^{3}$ \\
Fluid dynamic viscosity & $0.001 \mathrm{~Pa}{ }^{*} \mathrm{~s}$ \\
DEM time step size & $1.0 \mathrm{E}-7 \mathrm{~s}$ \\
CFD time step size & $5.0 \mathrm{E}-4 \mathrm{~s}$ \\
Time step for coupling & $1.0 \mathrm{E}-3 \mathrm{~s}$ \\
Simulation time & $5.0 \mathrm{~s}$ \\
\hline
\end{tabular}




\section{List of Figures:}

Figure 1. Contact models among particles.

Figure 2. Schematic diagram of implementing coupled CFD-DEM model.

Figure 3. Simulation of free settling experiments in water.

Figure 4. Comparison of settling velocity in different models.

Figure 5. Pipe flow soil erosion model.

Figure 6. Comparison of flow and particle velocities at t = 5.0 s: (a) Model A; (b) Model B;

(c) Model C.

Figure 7. Porosity contour graph of sandpile at t= $5.0 \mathrm{~s}$ : (a) Model A: (b) Model B; (c)

Model C.

Figure 8. Flow velocity along the cross section of $X=0.125 \mathrm{~m}$ at $\mathrm{t}=5.0 \mathrm{~s}$.

Figure 9. Development of flow velocity on the surface of sandpile (height $=0.02 \mathrm{~m}$ ).

Figure 10. Contour graph of fluid pressure at $t=5.0 \mathrm{~s}$ : (a) Model A: (b) Model B; (c) Model C.

Figure 11. Pressure field along the cross section of $X=0.125 \mathrm{~m}$ at $\mathrm{t}=5.0 \mathrm{~s}$.

Figure 12. Development of fluid pressure on the sandpile surface (height $=0.02 \mathrm{~m}$ ).

Figure 13. Sand profiles before (a) and after 5-second erosion: (b) Model A; (c) Model B; (d) Model C.

Figure 14. Distribution of drag force in sand profile at $t=5.0 \mathrm{~s}$ : (a) Model A; (b) Model B; (c) Model C.

Figure 15. Velocity distribution of sand particles in different models at $t=5.0 \mathrm{~s}$.

Figure 16. Translational kinetic energy of the whole sand aggregates.

Figure 17. Rotational kinetic energy of the whole sand aggregates.

Figure 18. Total (translational + rotational) kinetic energy of the whole sand aggregates. 


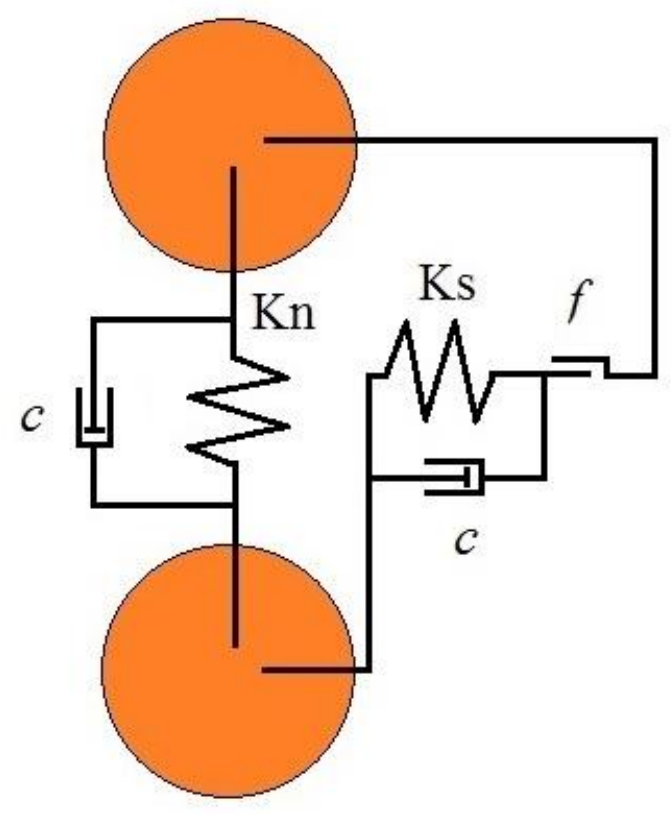

Figure 1. Contact models among particles. 


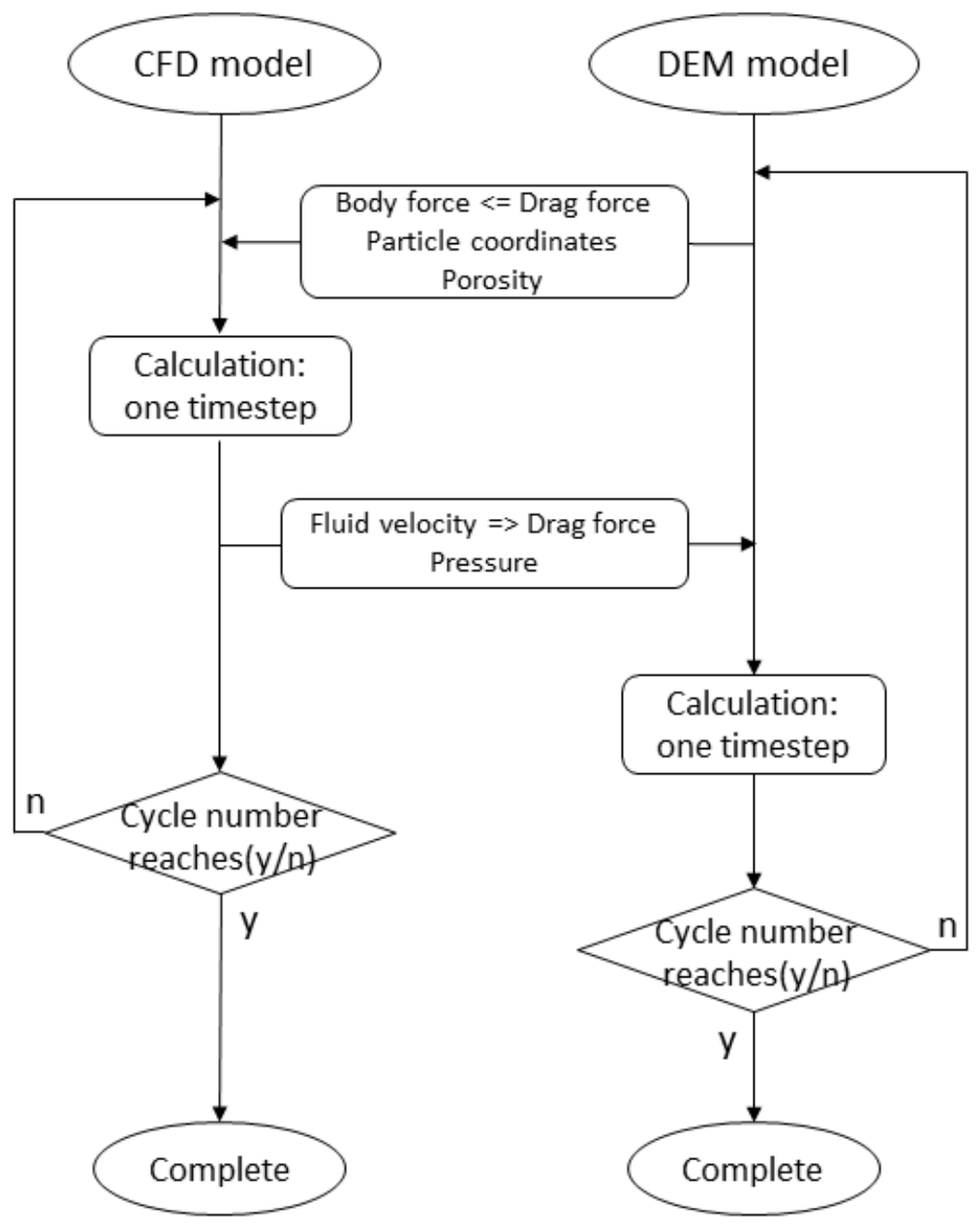

Figure 2. Schematic diagram of implementing coupled CFD-DEM model. 


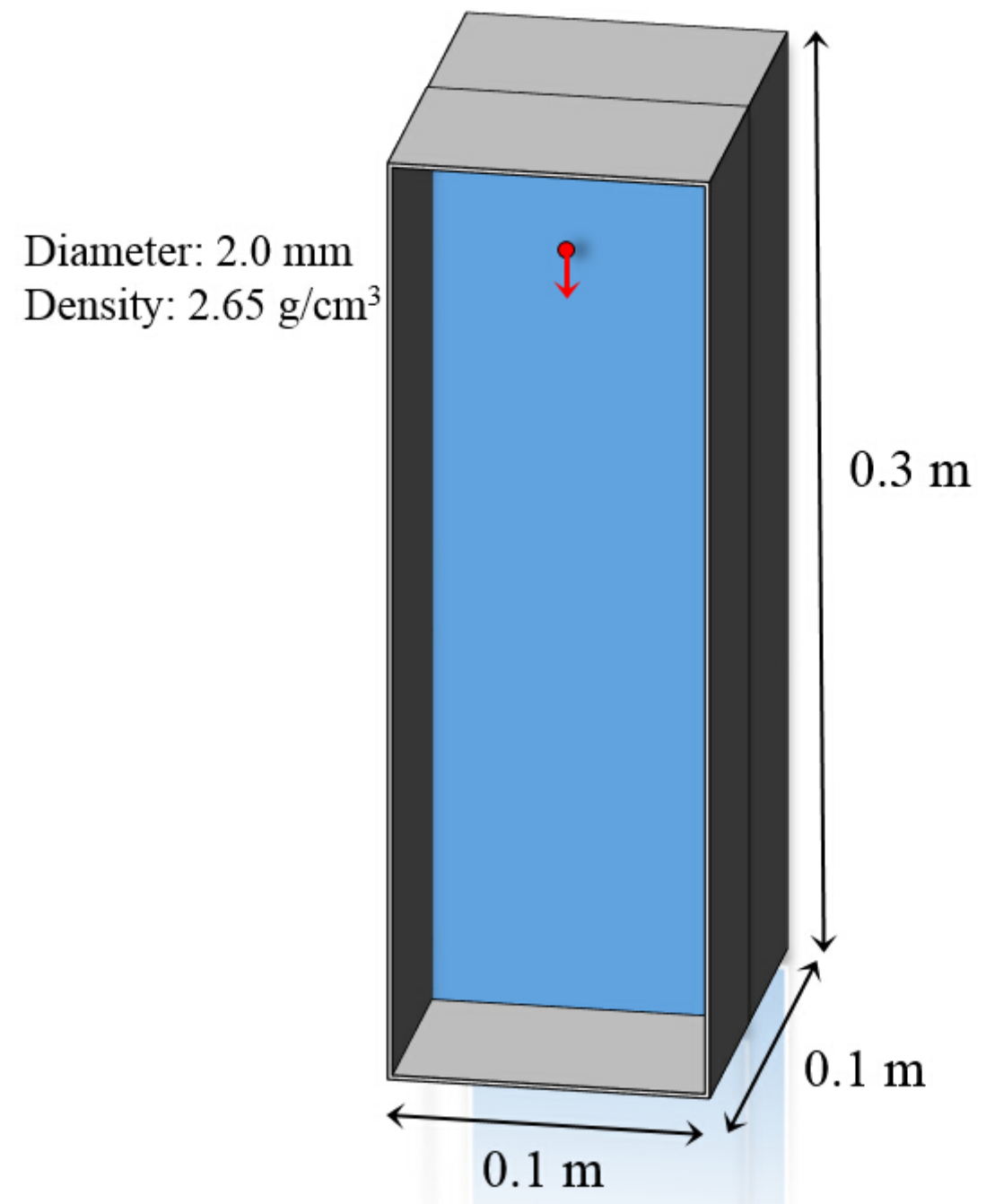

Figure 3. Simulation of free settling experiments in water. 


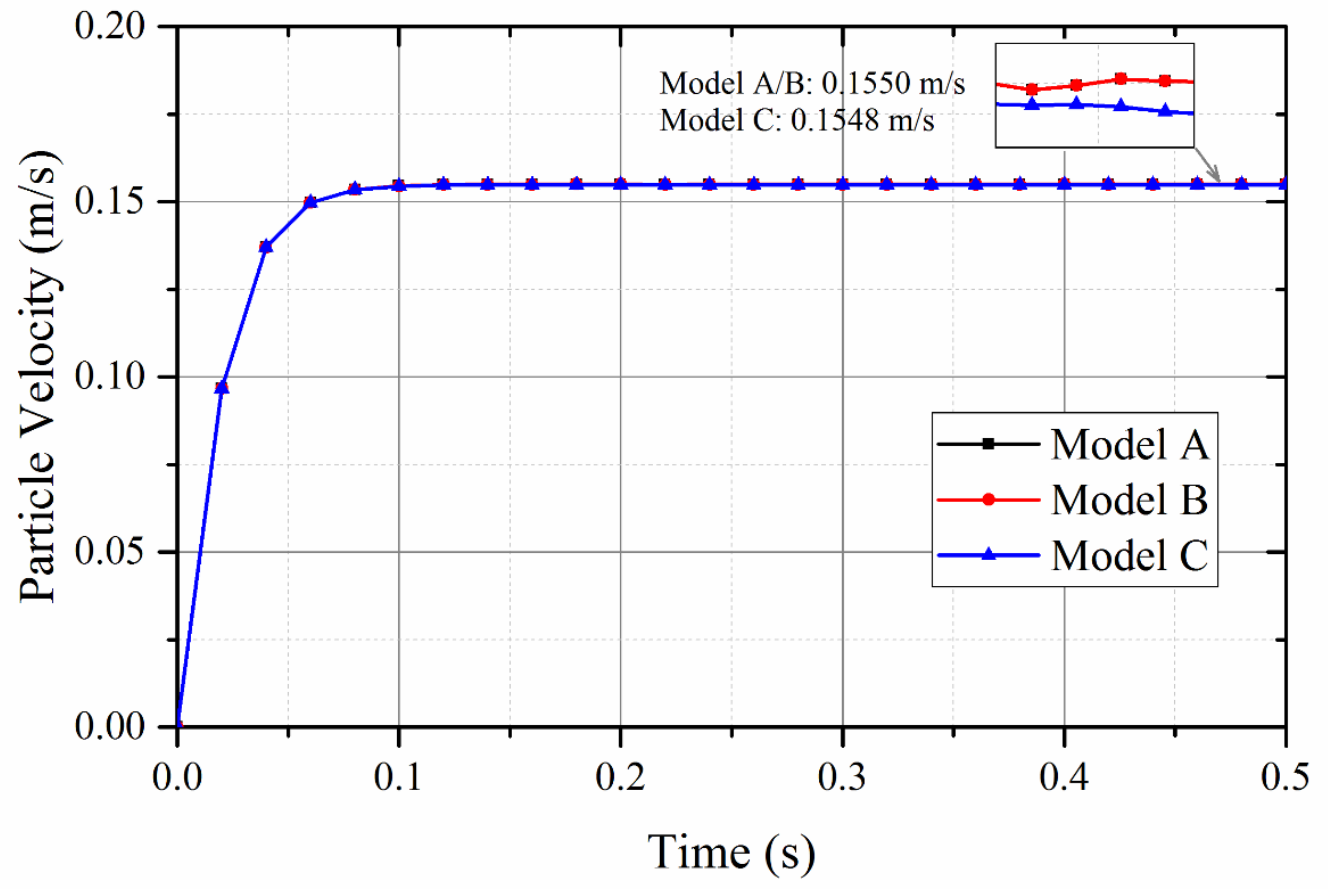

Figure 4. Comparison of settling velocity in different models. 


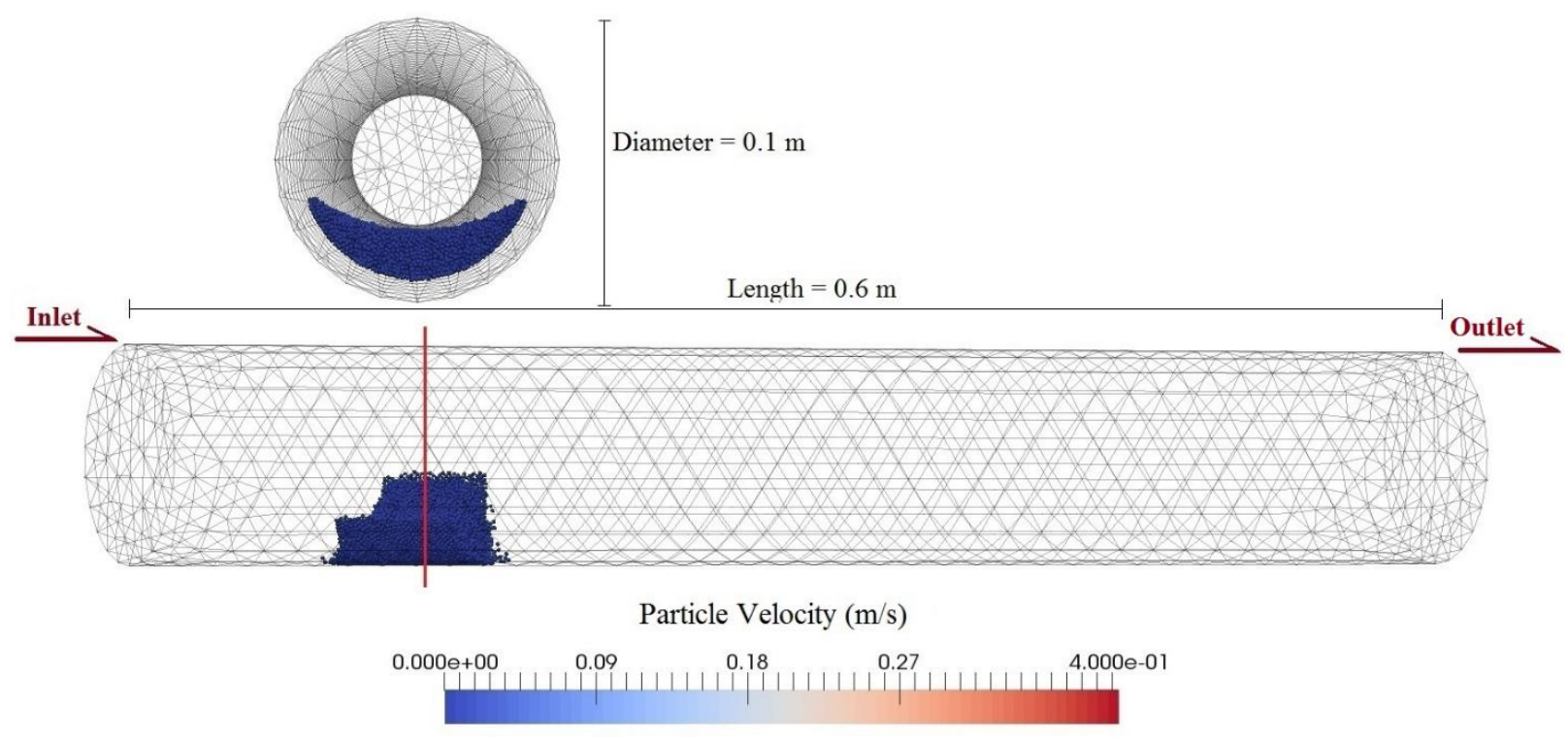

Figure 5. Pipe flow soil erosion model. 
(a)

Fluid Velocity $(\mathrm{m} / \mathrm{s})$

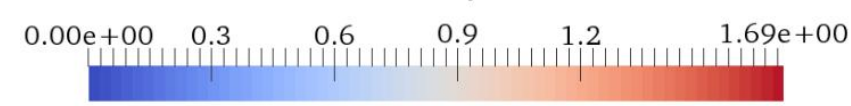

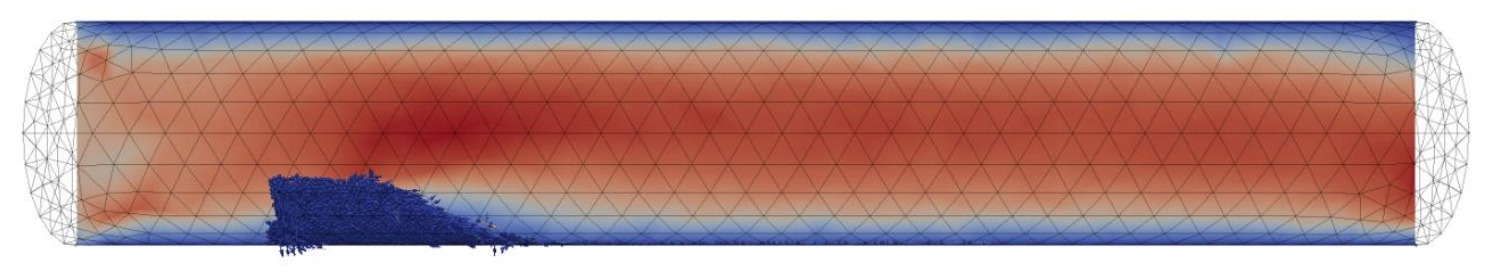

Particle Velocity $(\mathrm{m} / \mathrm{s})$

$0.00 \mathrm{e}+00 \quad 0.1,0.20 .32 \mathrm{e}-01$

(b)

Fluid Velocity $(\mathrm{m} / \mathrm{s})$

$0.00 e+00 \quad 0.3$
$\omega$

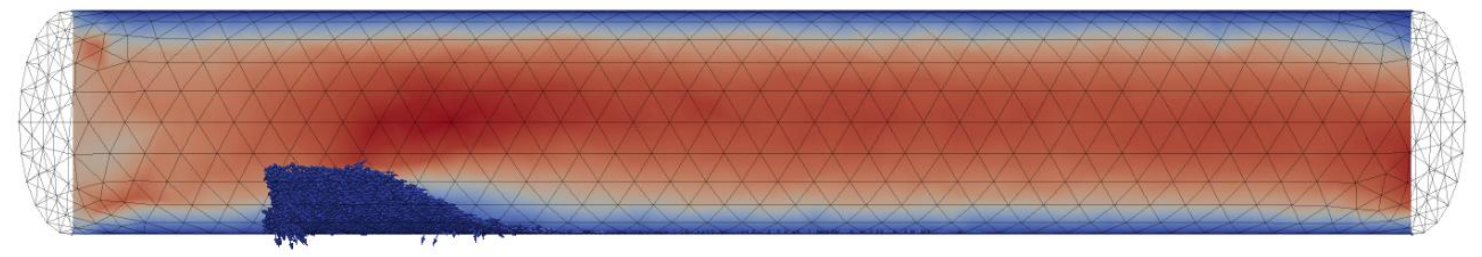

Particle Velocity $(\mathrm{m} / \mathrm{s})$

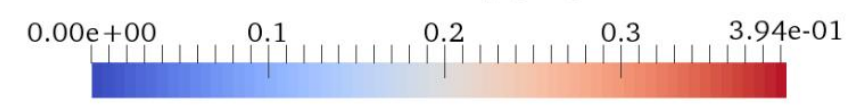


(c)

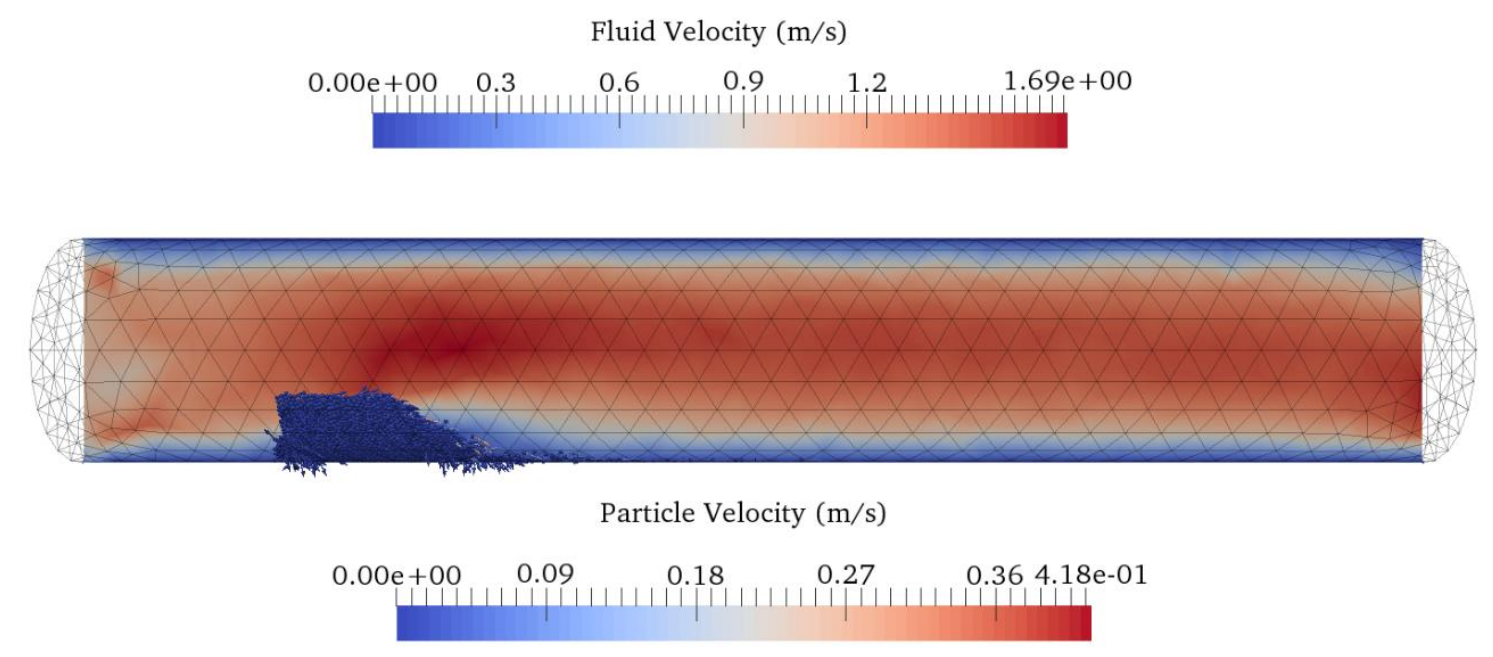

Figure 6. Comparison of flow and particle velocities at t = 5.0 s: (a) Model A; (b) Model B;

(c) Model C. 
(a)

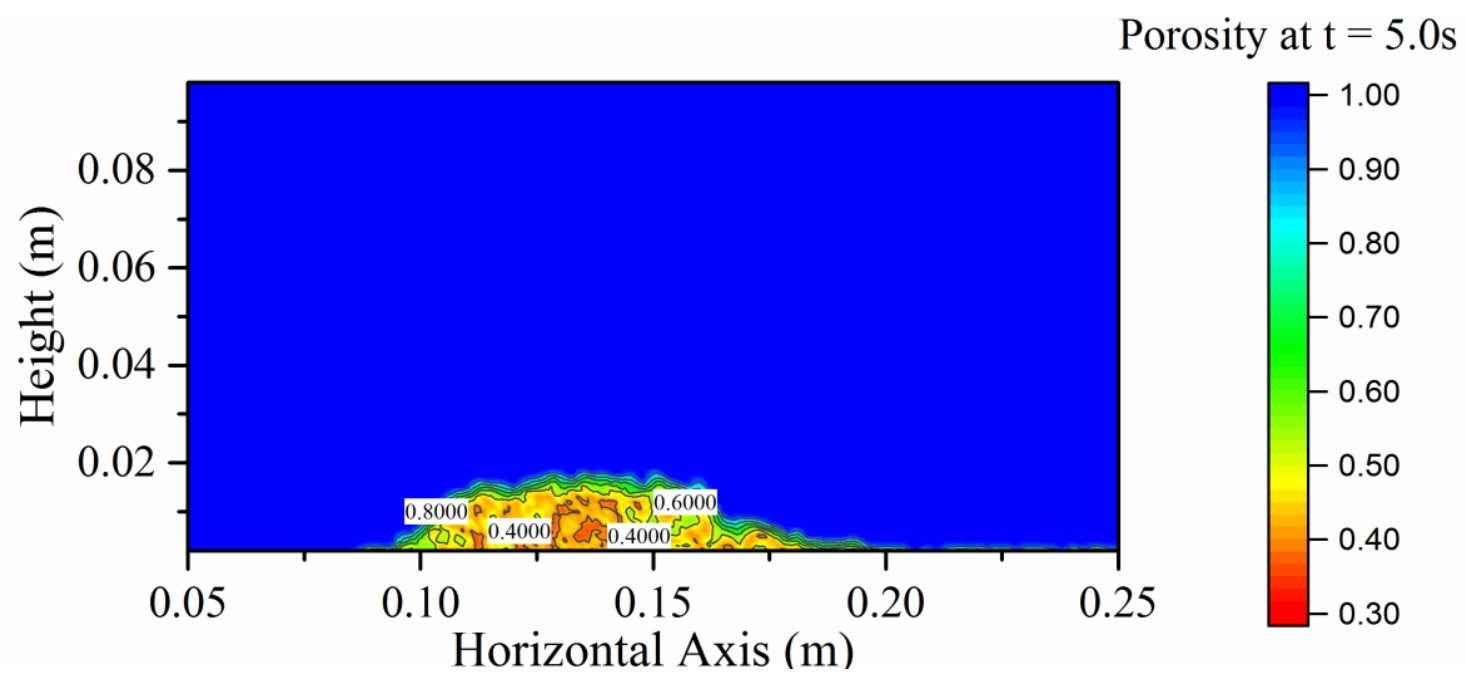

(b)

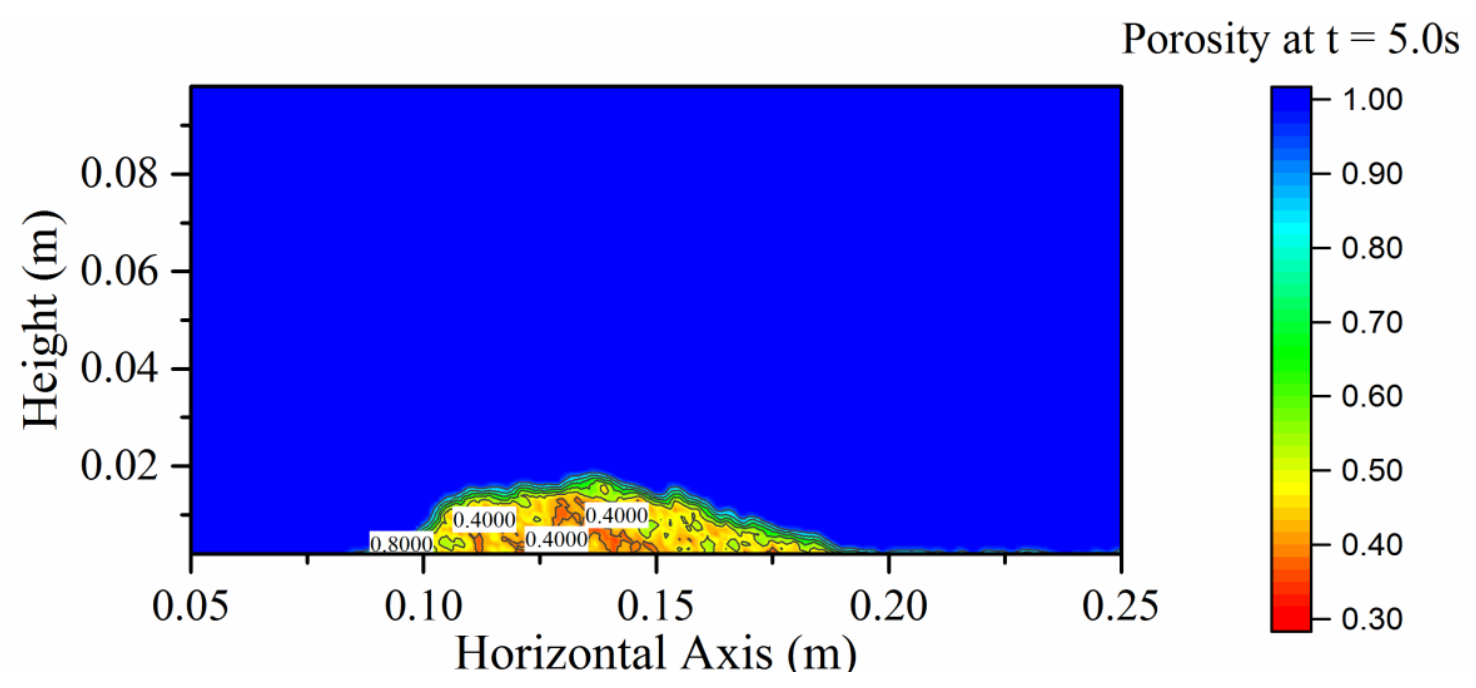


(c)

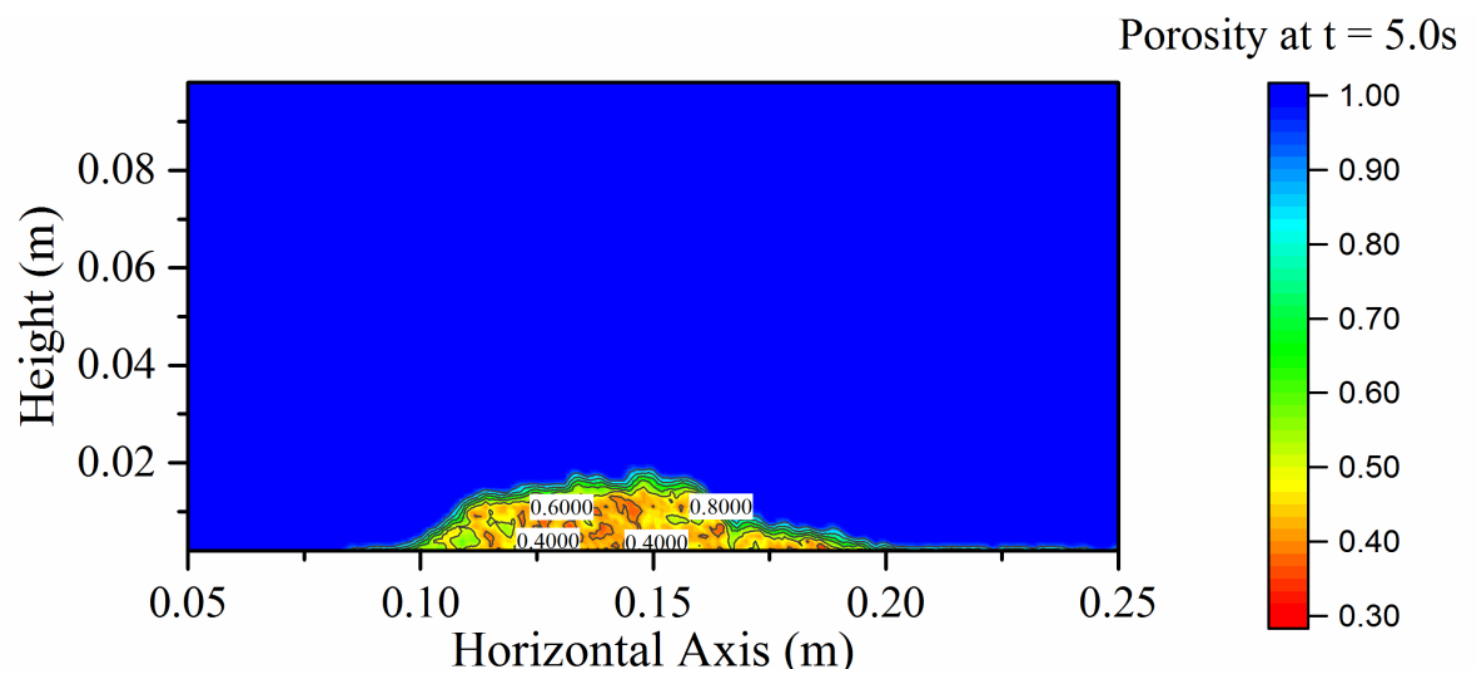

Figure 7. Porosity contour graph of sandpile at $t=5.0$ s: (a) Model A: (b) Model B; (c) Model C. 


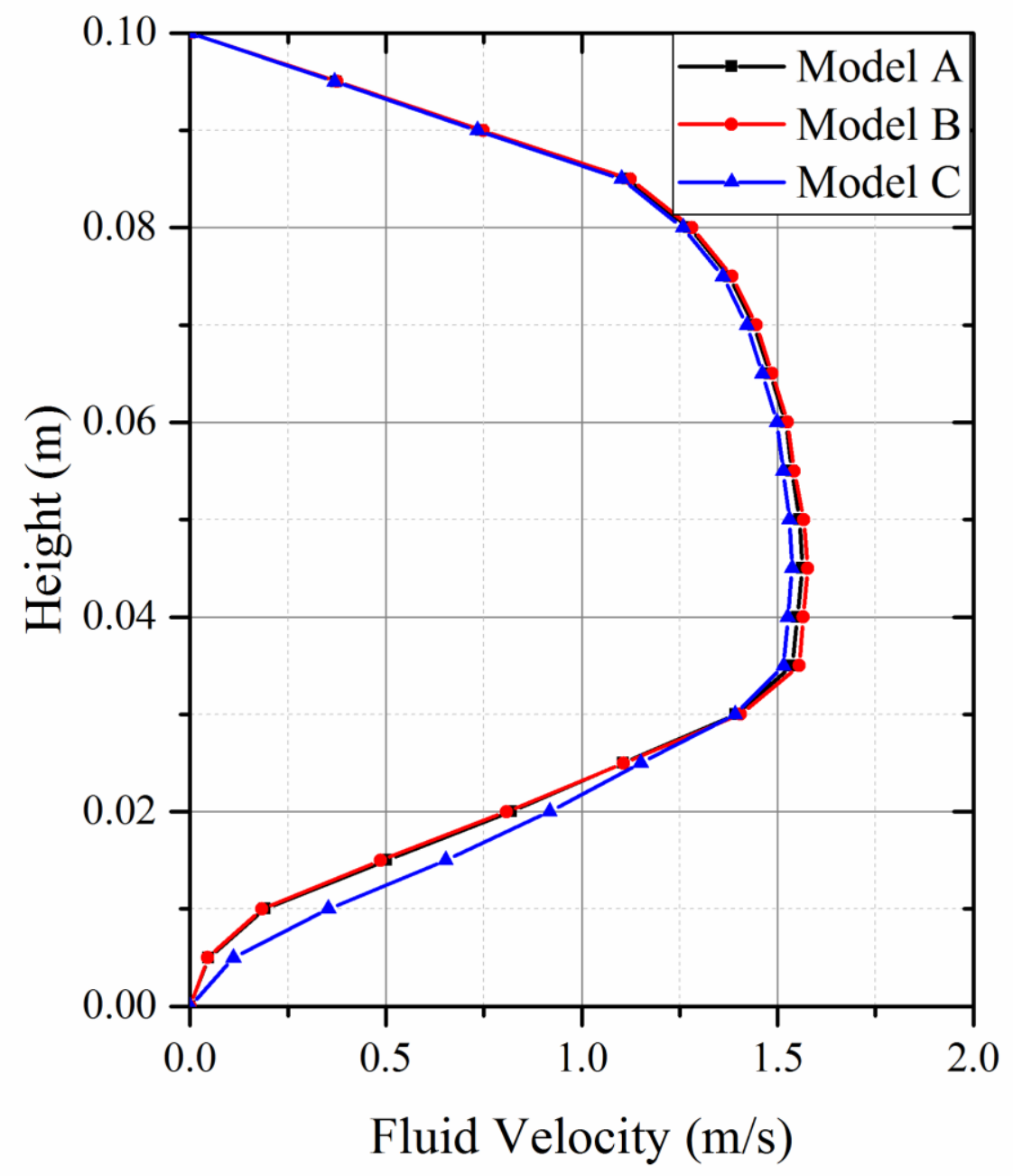

Figure 8. Flow velocity along the cross section of $X=0.125 \mathrm{~m}$ at $\mathrm{t}=5.0 \mathrm{~s}$. 


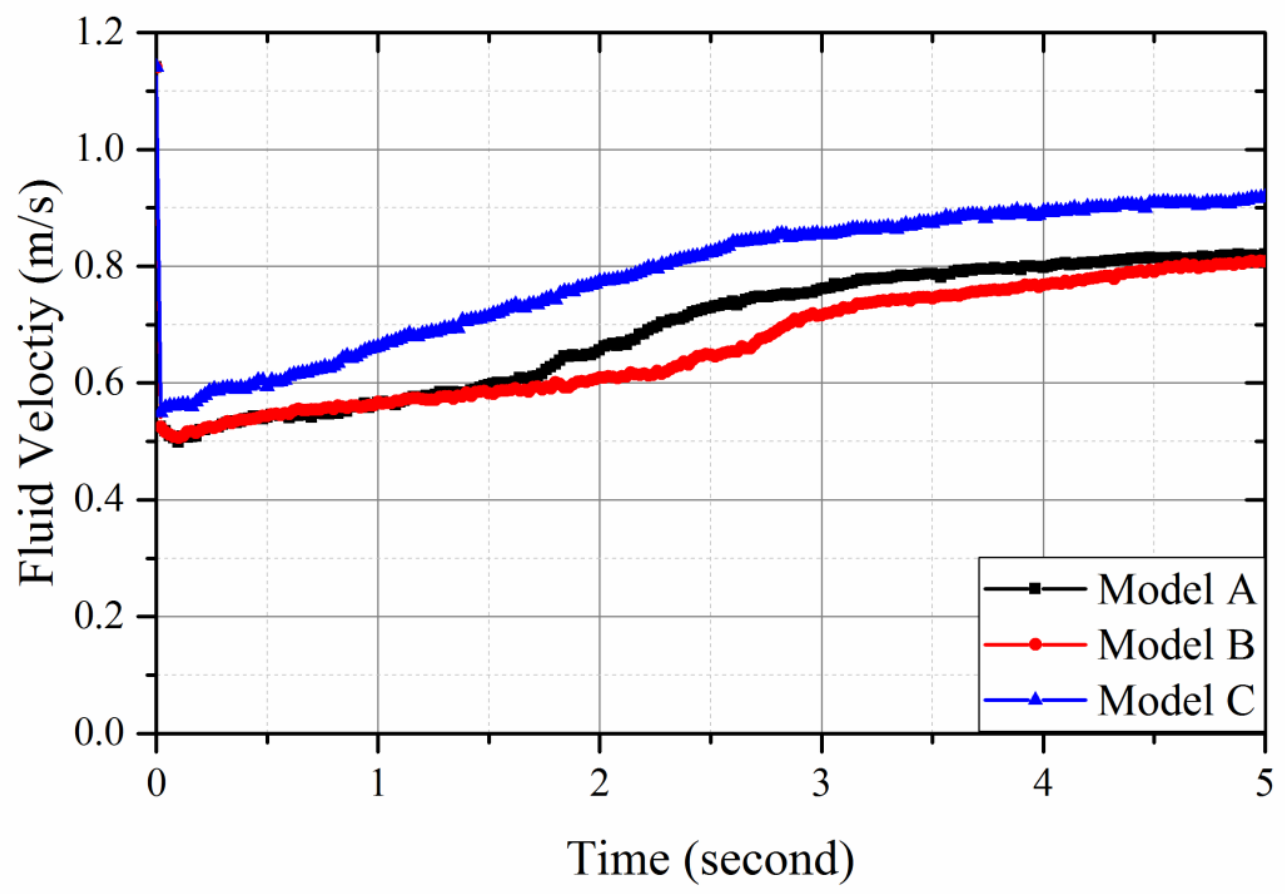

Figure 9. Development of flow velocity on the surface of sandpile (height $=0.02 \mathrm{~m}$ ). 
(a)

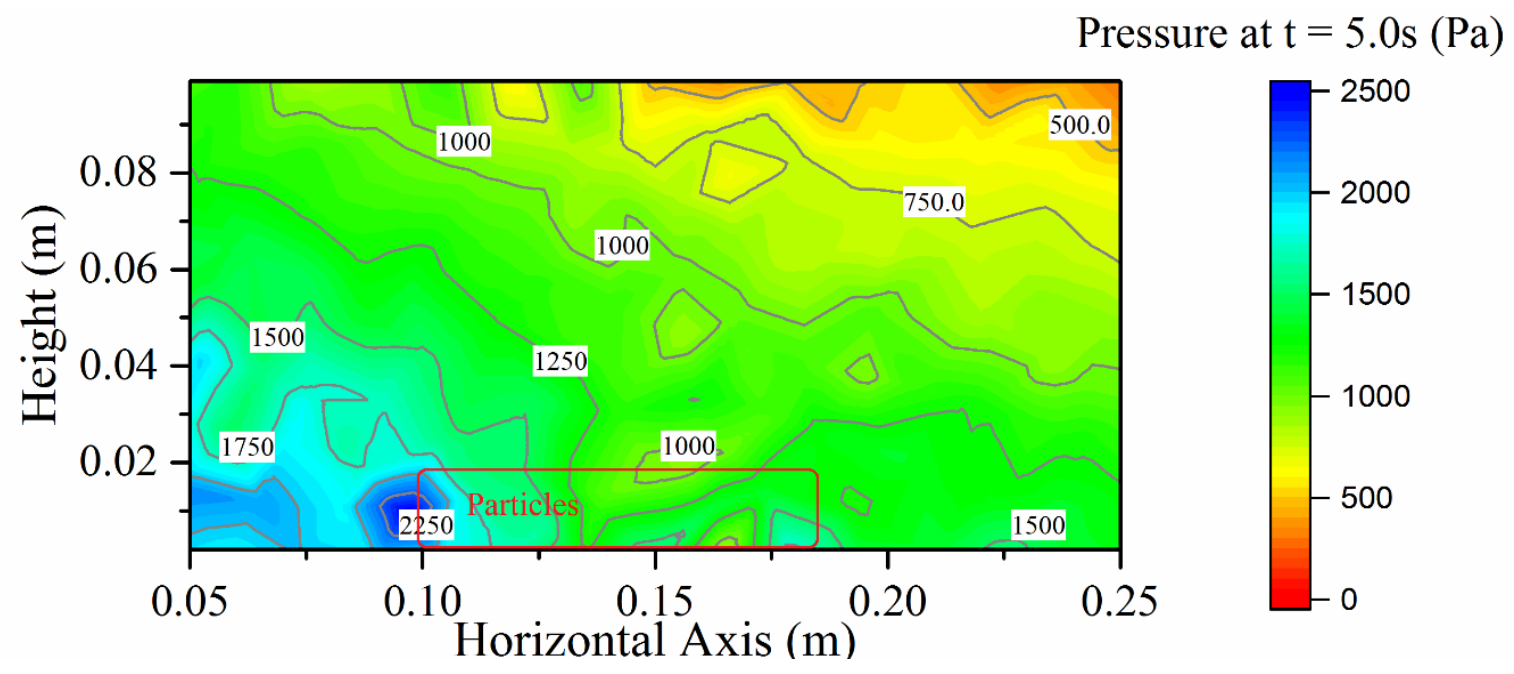

(b)

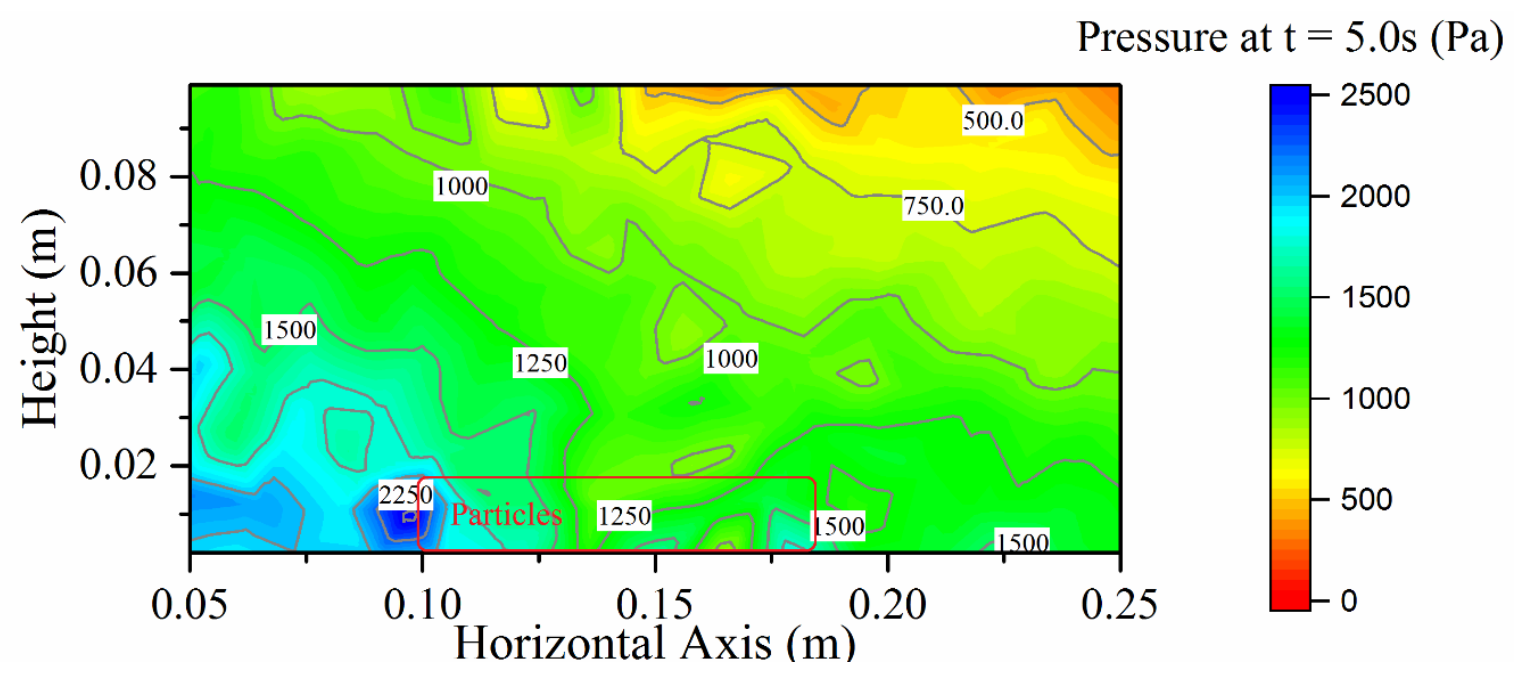


(c)

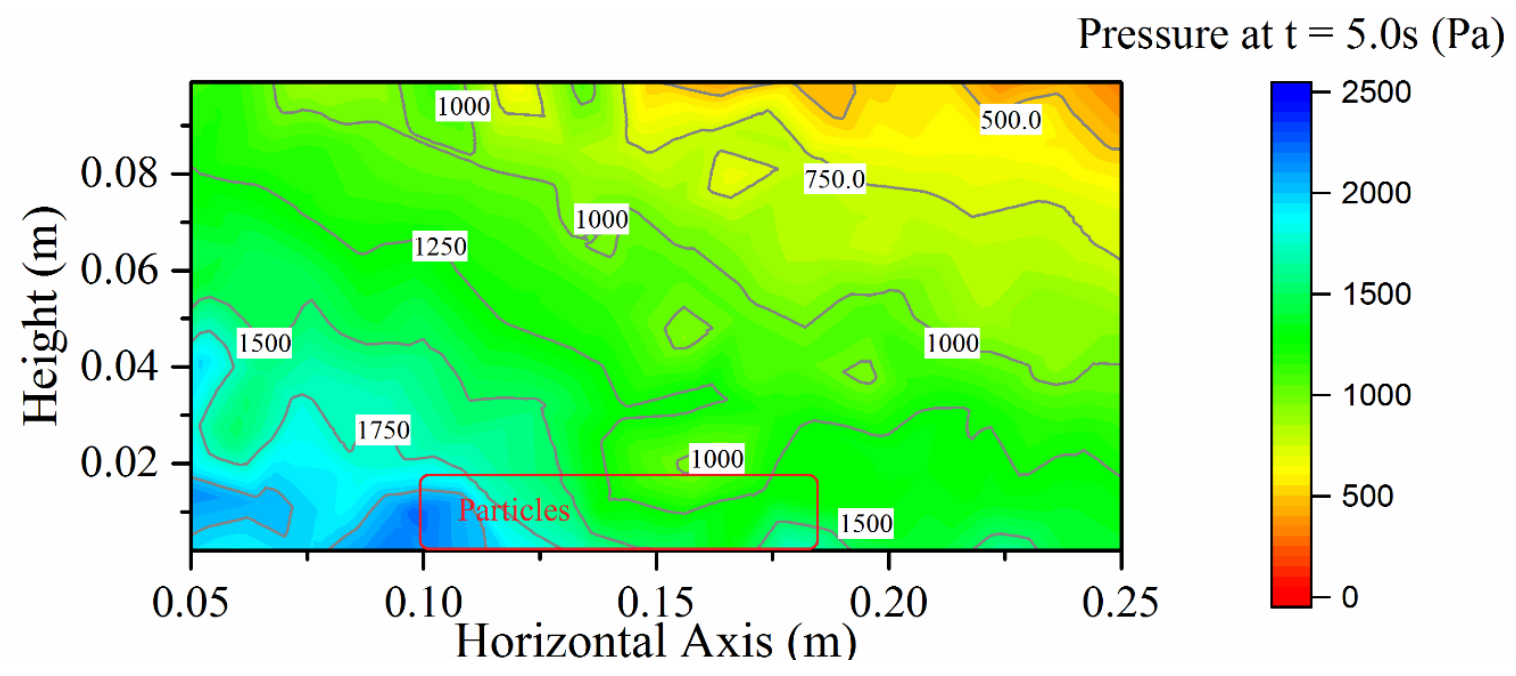

Figure 10. Contour graph of fluid pressure at t $=5.0 \mathrm{~s}$ : (a) Model A: (b) Model B; (c) Model

C. 


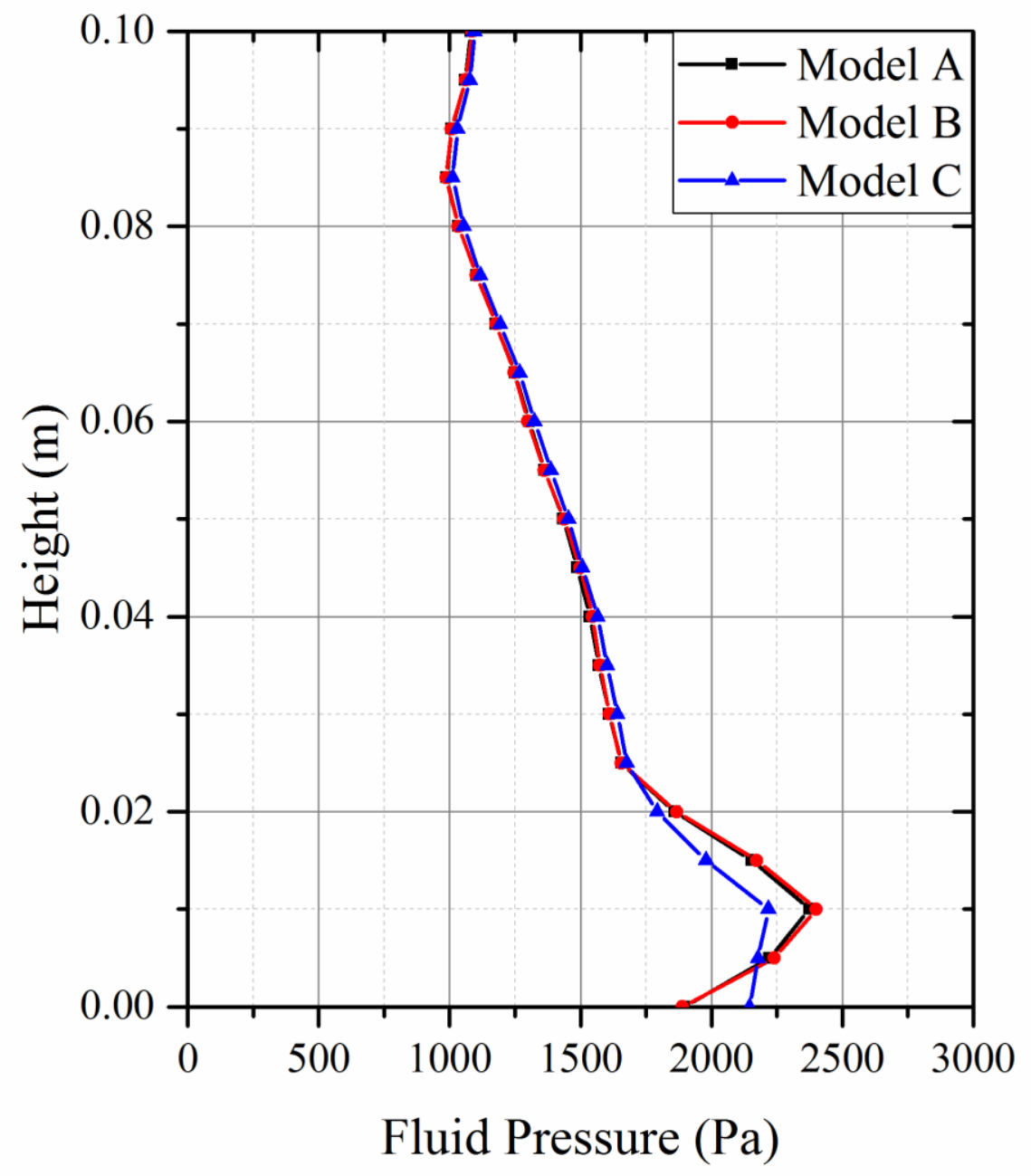

Figure 11. Pressure field along the cross section of $X=0.125 \mathrm{~m}$ at $t=5.0 \mathrm{~s}$. 


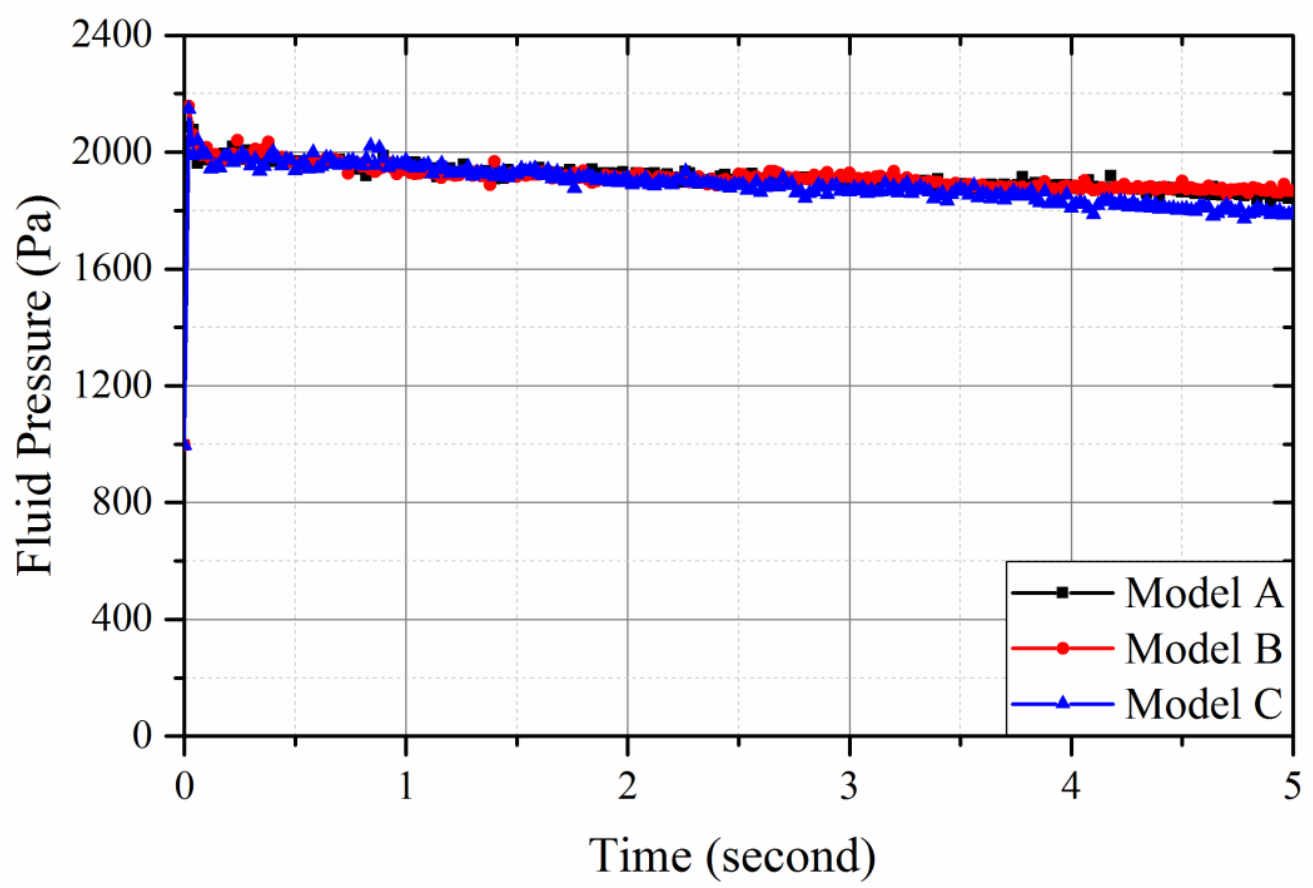

Figure 12. Development of fluid pressure on the sandpile surface (height $=0.02 \mathrm{~m})$. 
(a)

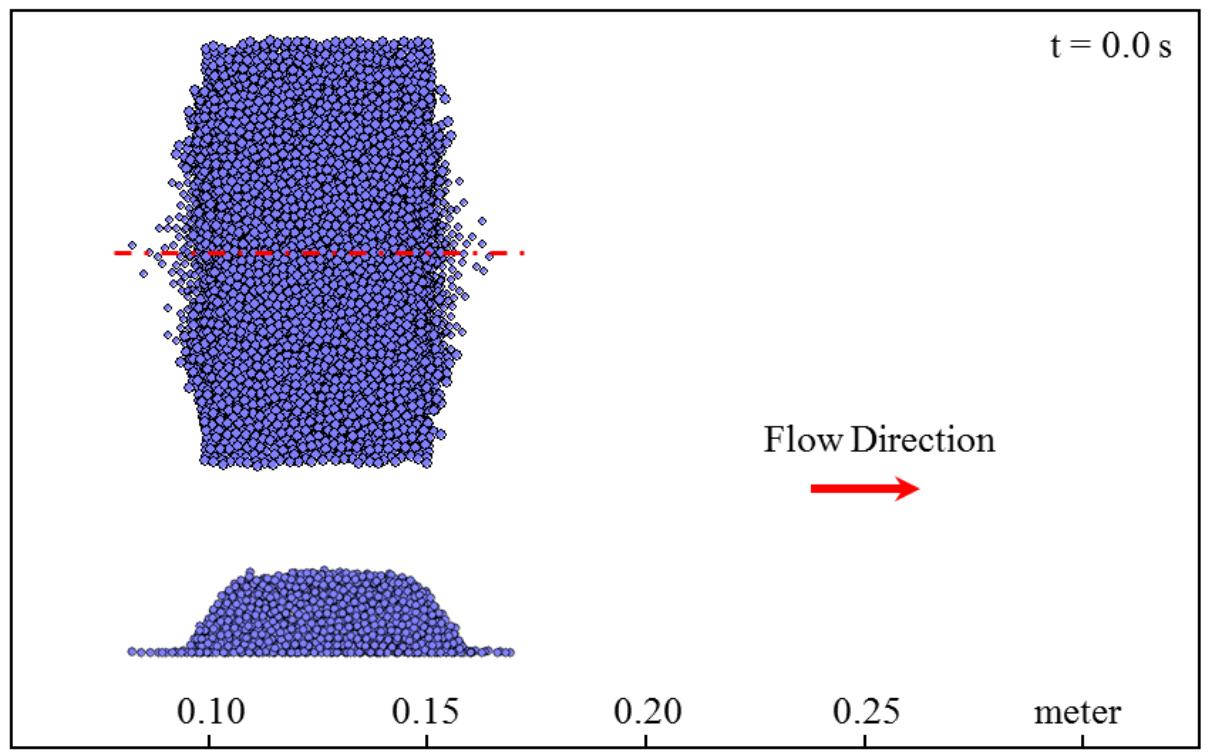

(b)

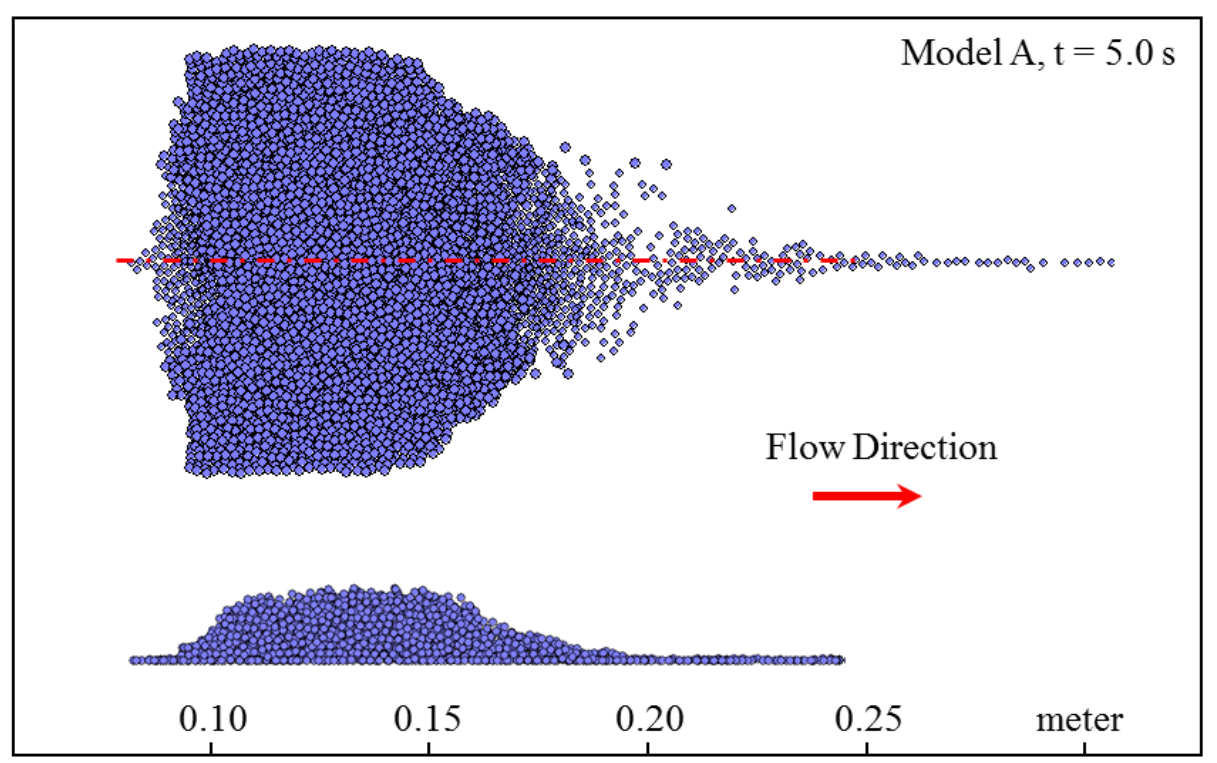

(c) 


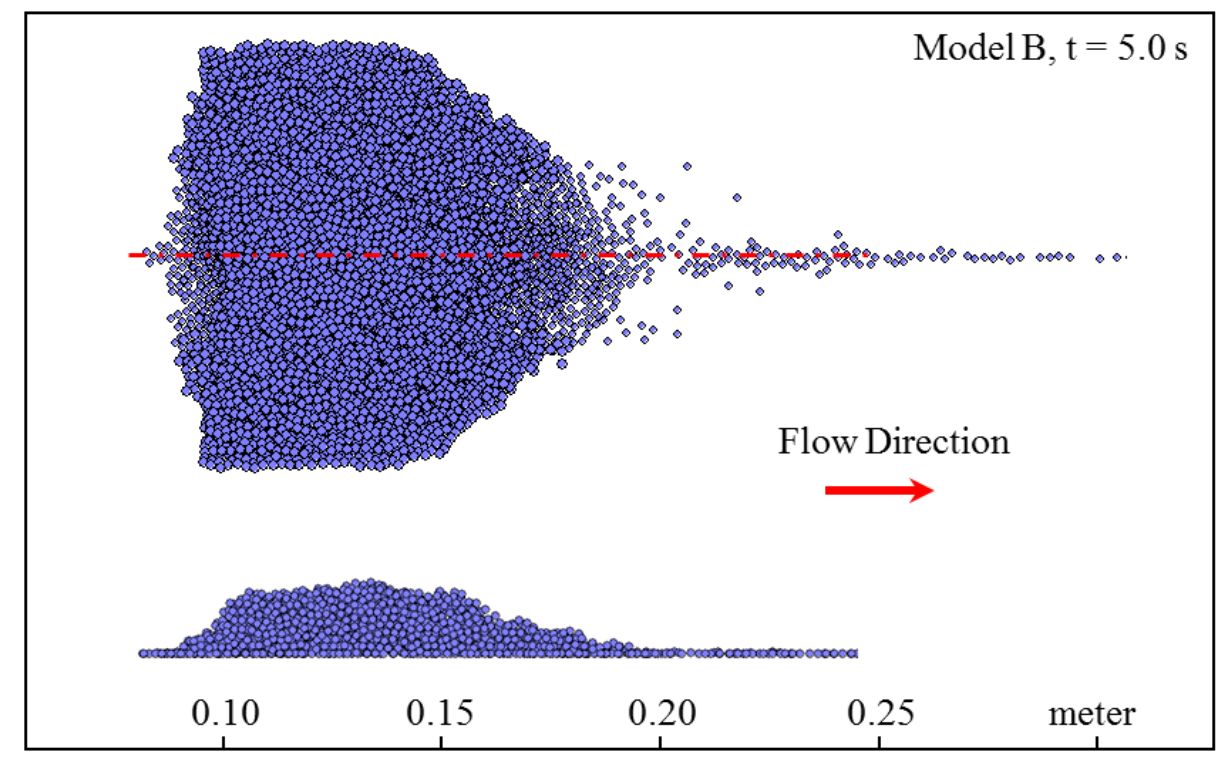

(d)

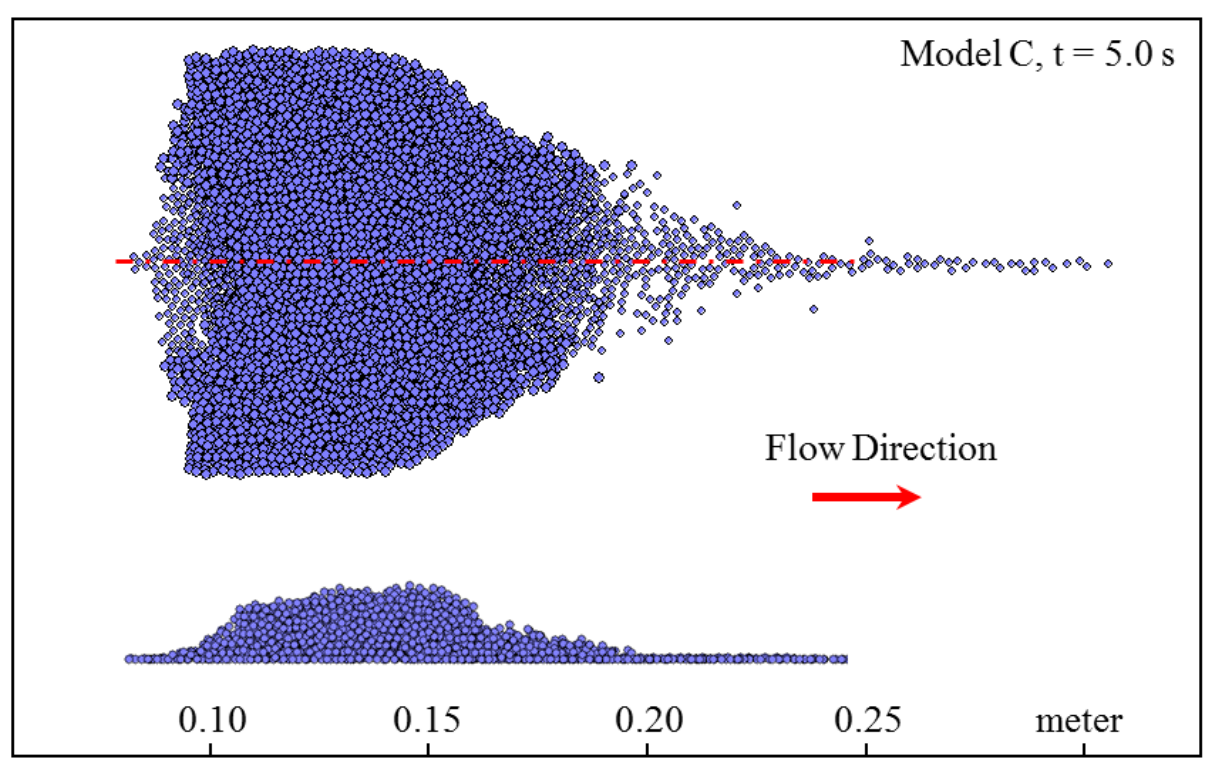

Figure 13. Sand profiles before (a) and after 5-second erosion: (b) Model A; (c) Model B; (d) Model C. 
(a)

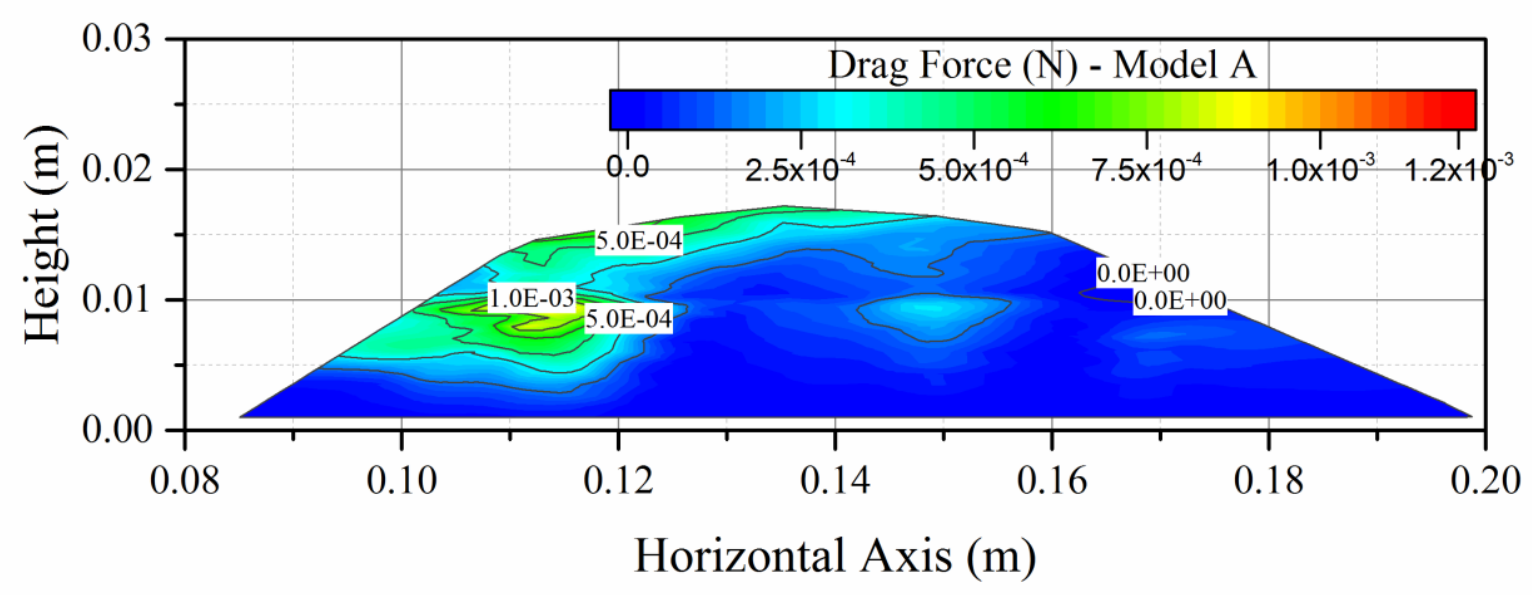

(b)

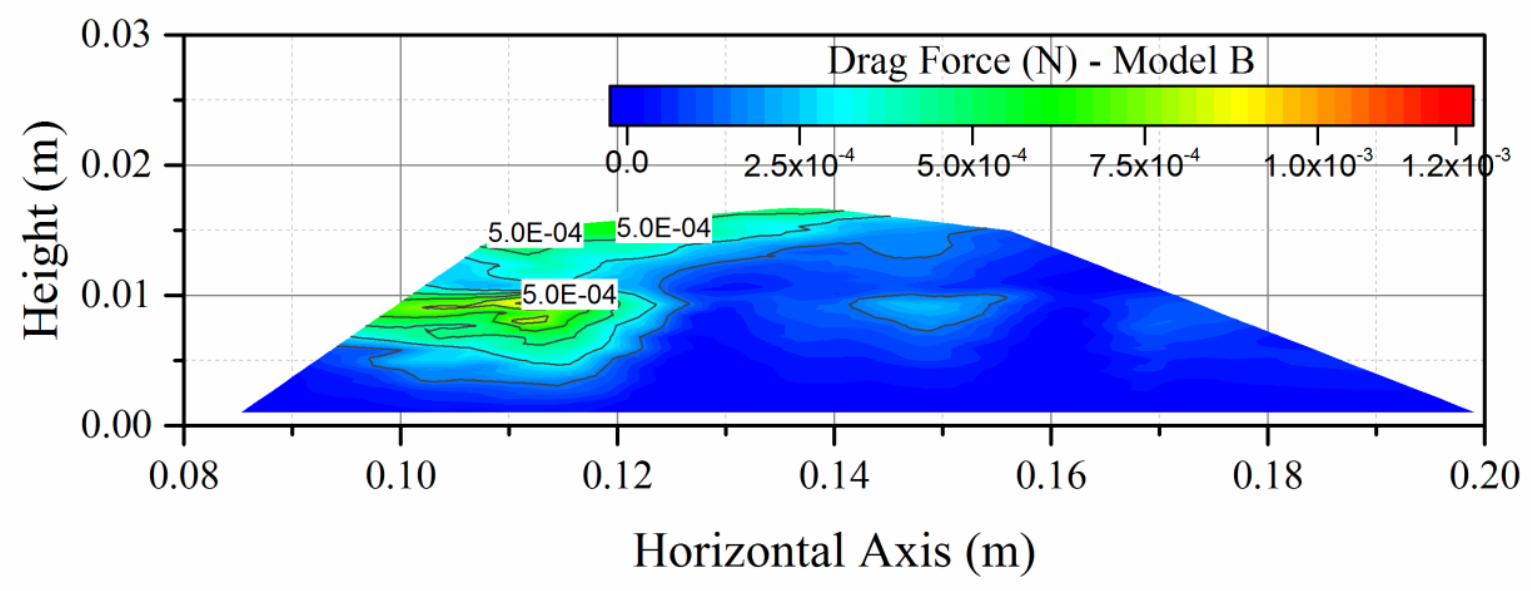


(c)

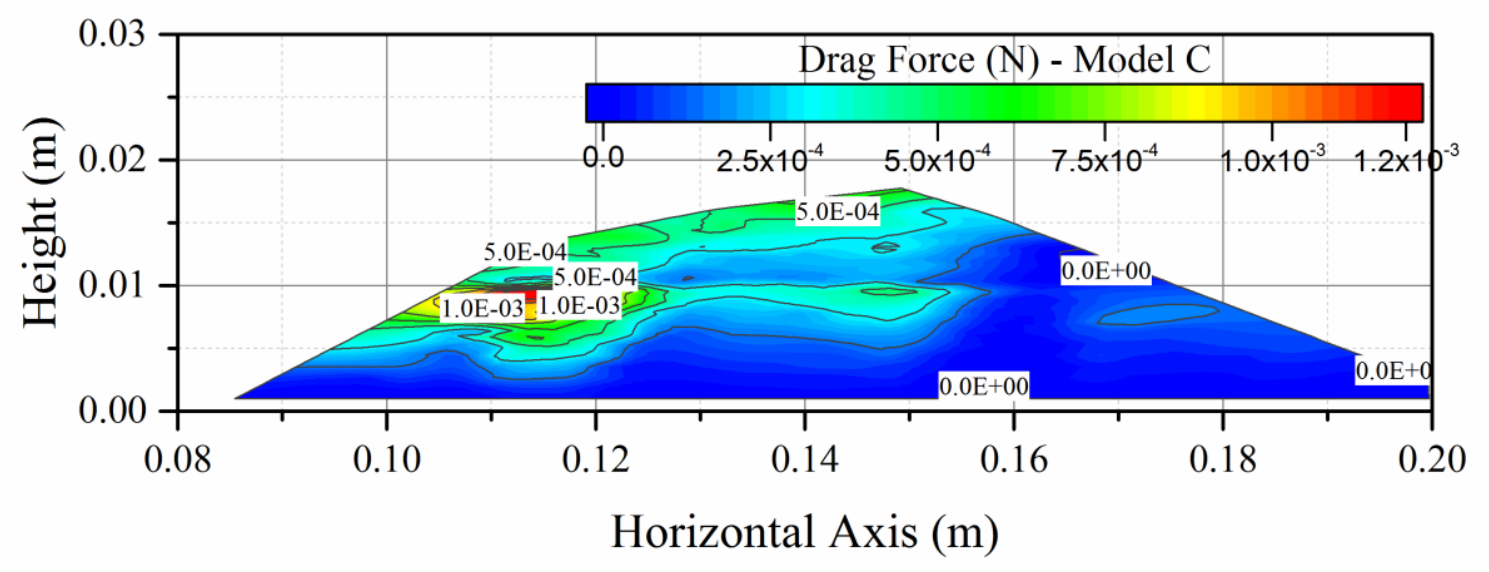

Figure 14. Distribution of drag force in sand profile at $t=5.0 \mathrm{~s}$ : (a) Model A; (b) Model B; (c) Model C. 


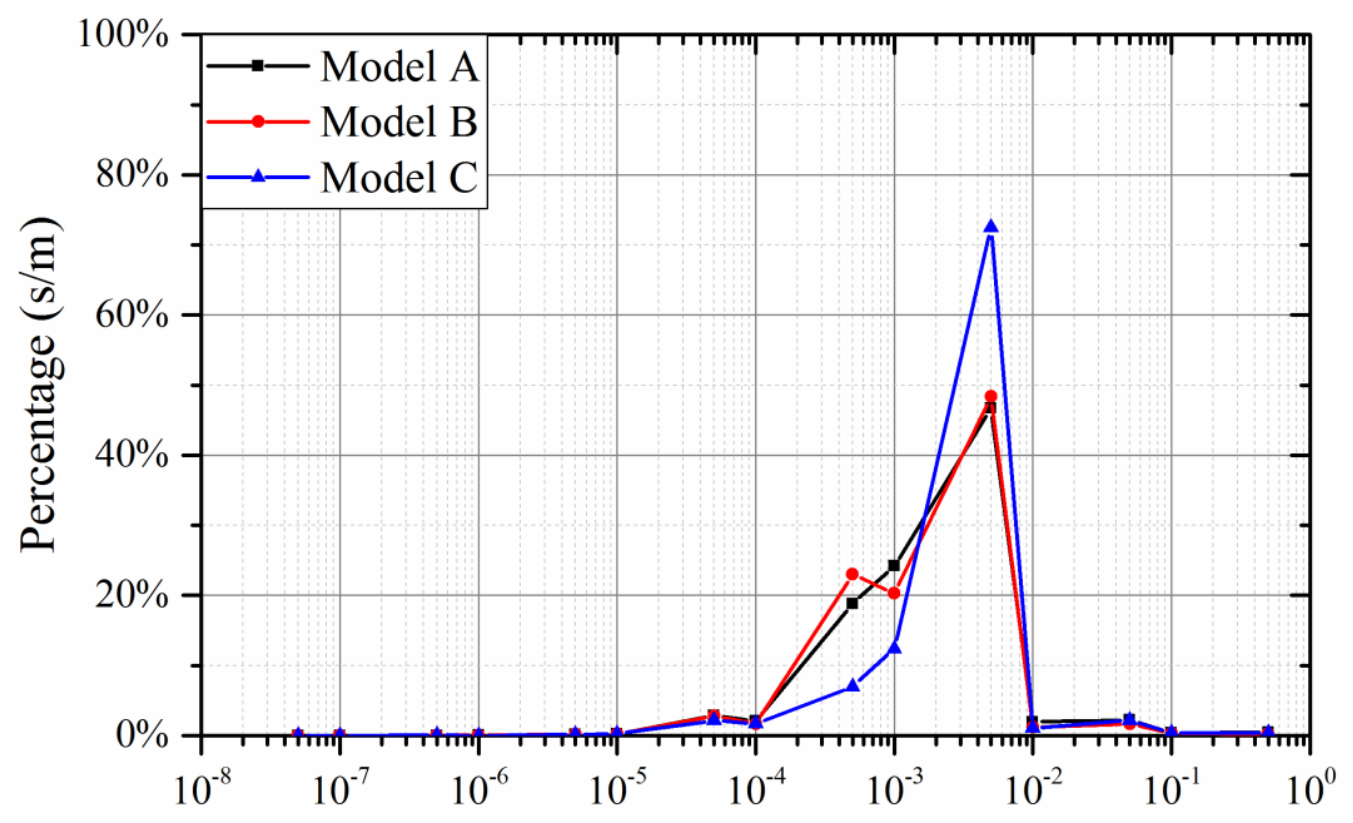

Figure 15. Velocity distribution of sand particles in different models at $t=5.0 \mathrm{~s}$. 


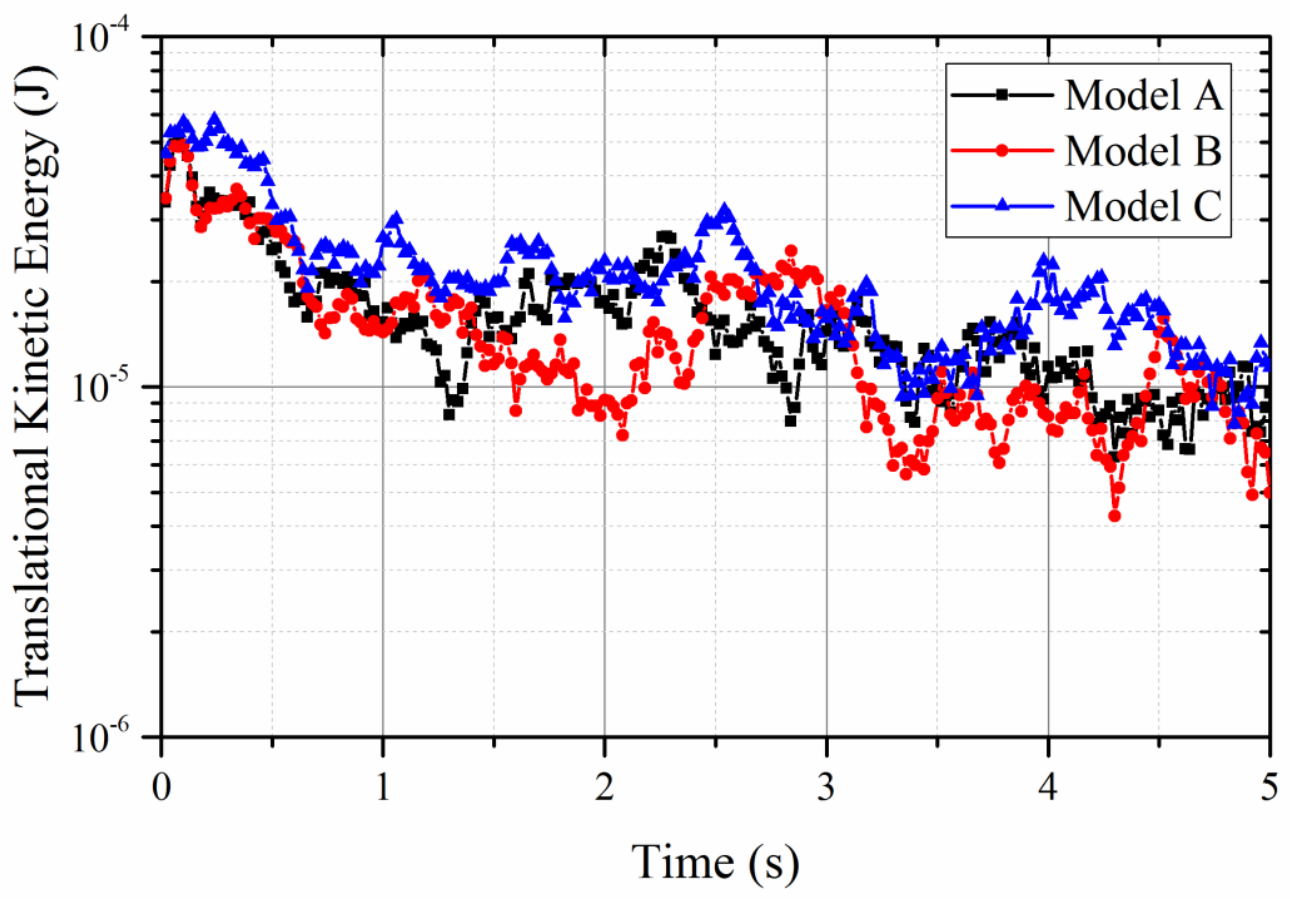

Figure 16. Translational kinetic energy of the whole sand aggregates. 


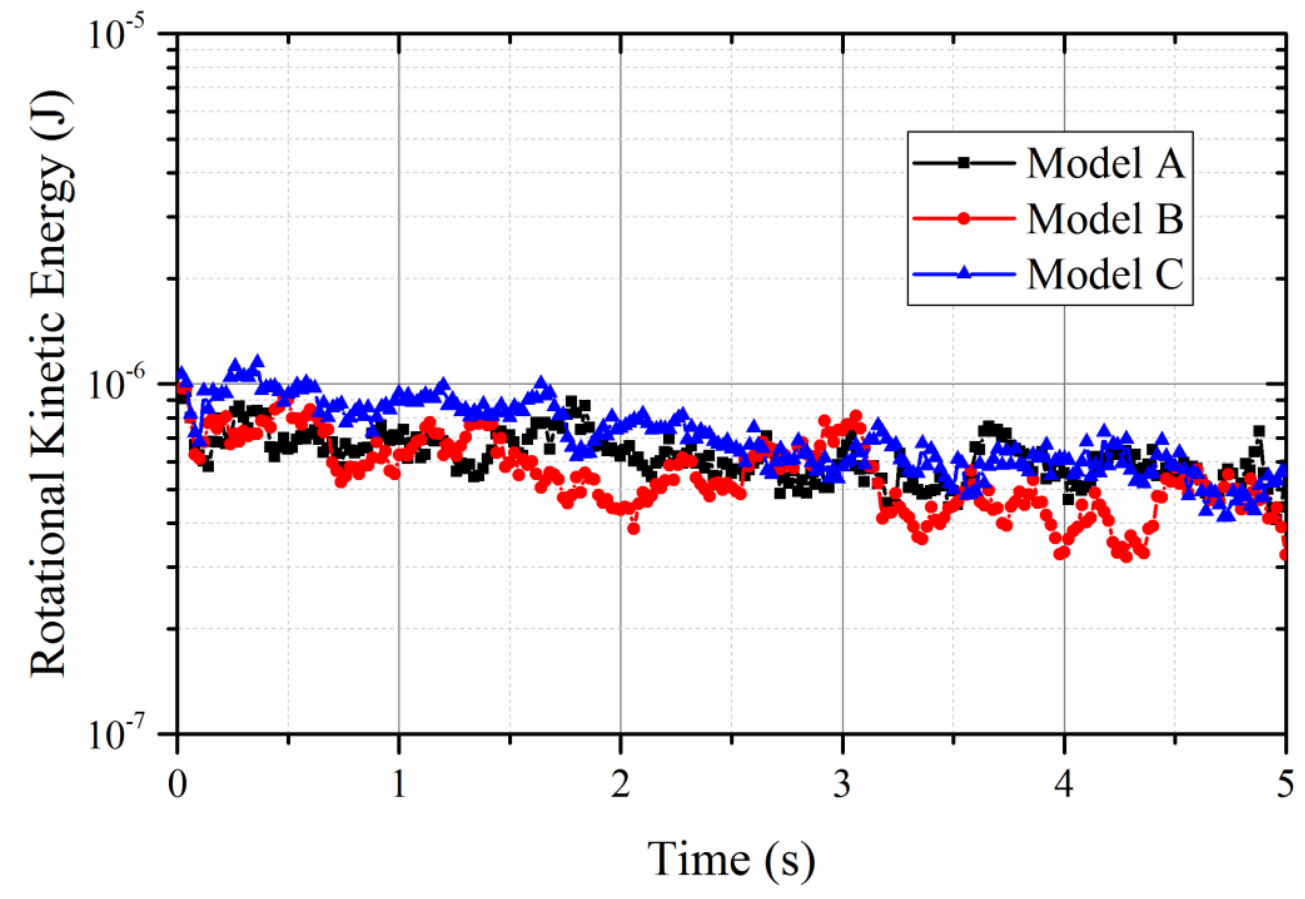

Figure 17. Rotational kinetic energy of the whole sand aggregates. 


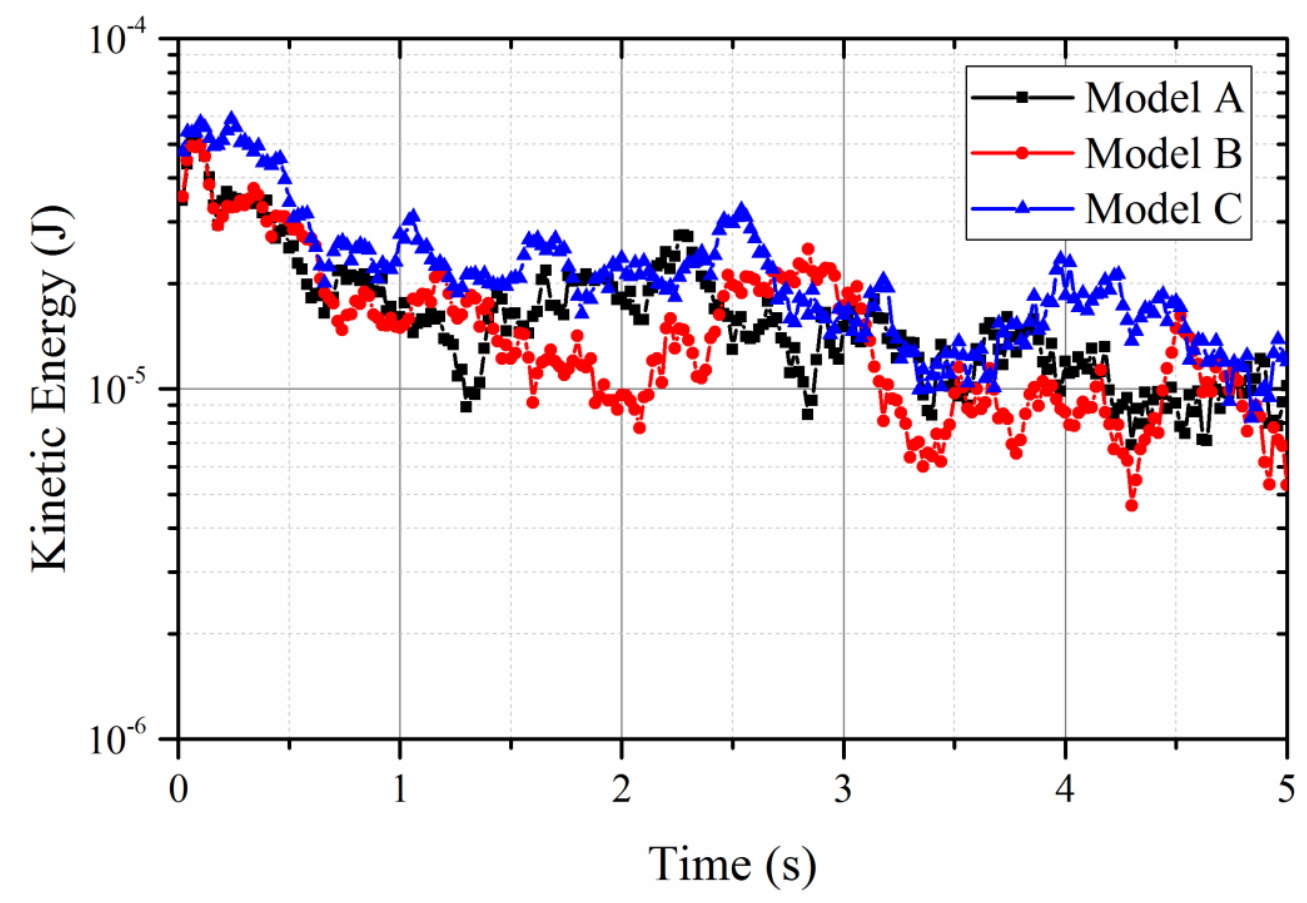

Figure 18. Total (translational + rotational) kinetic energy of the whole sand aggregates. 